\title{
Generating Cluster Formulas Using The Primary Clusters And The K(n) Parameters
}

\author{
Enos Masheija Rwantale Kiremire
}

Correspondence: Enos Masheija Rwantale Kiremire, Professor of Chemistry, University of Namibia. Hochland Park, P. O. Box, 98013, Pelican Square, Tauben Glen, Windhoek, Namibia. E-mail: kiremire15@yahoo.com

Received: January 27, 2018 Accepted: April 10, 2018 Online Published: April 27, 2018

doi:10.5539/ijc.v10n2p85

URL: https://doi.org/10.5539/ijc.v10n2p85

\begin{abstract}
The formulas of transition metal clusters can be regarded as multiples of their respective PRIMARY CLUSTERS and $\mathrm{K}(\mathrm{n})$ parameters. A primary cluster of a transition metal skeletal element can be defined as that cluster of a mono-skeletal element which obeys the 18 electron rule. Such clusters, among others include, $\mathrm{Cr}(\mathrm{CO})_{6}, \mathrm{Fe}(\mathrm{CO})_{5}$, $\mathrm{Ni}(\mathrm{CO})_{4}$, and $\mathrm{Zn}(\mathrm{CO})_{3}$ and the respective $\mathrm{K}$ values of the skeletal elements are 6, 5, 4 and 3. The selected $\mathrm{K}(\mathrm{n})$ series are given and the derived hypothetical golden clusters are given as examples for illustrations. Selected known golden clusters are also found to be multiples of $\mathrm{K}(\mathrm{n})$ parameters and the 18 valence fragment cluster, AuL 3.5 . The graphical representations of a few selected examples of golden clusters are given.
\end{abstract}

Keywords: primary cluster, skeletal element, k(n) series, torroidal, spherical

\section{Introduction}

Cluster chemistry covers a wide range of clusters. Among others, It includes those which are solely centered around boron[B] skeletal element(boranes) (Lipscomb, 1963; Fox \& Wade, 2003; Jemmis, 2005, Jemmis, et al, 2008; Kiremire, 2014a, boron-carbon[B-C] skeletal elements) carboranes (Greenwood \& Earnshaw, 1998; Housecroft \& Sharpe, 2012), boron-transition metal [B-M] (metalloboranes (Kiremire, 2016a) boron-carbon-metal[B-C-M](metallocarboranes),metal-carbonyl[M-CO]transition metal carbonyls(Kiremire, 2017a) Zintl ion clusters (2016b) and gold-phosphorus[Au-P] clusters(Mingos, 1984, Kiremire, 2016c, 2017b). The 4n series method has been found extremely useful in analyzing all these clusters along side Wade-Mingos rules (Wade, 1971; Mingos 1972). Using the 4n series method, it has been found that the multiples of [BH] fragments comprising of 4 electron valence content can applied to generate all the known formulas of borane clusters while the 14 electron valence electron content fragments such as $\left[\mathrm{Os}(\mathrm{CO})_{3}\right]$ can be utilized to generate osmium carbonyl clusters (Kiremire,2016d,2016e). In this paper, it is intended to demonstrate that also using the [8] electron valence electron content Primary Clusters such as $\left[\mathrm{BH}_{5}\right],\left[\mathrm{CH}_{4}\right]$ can be utilized to generate borane and hydrocarbon clusters respectively while the [18] electron valence content primary clusters such as $\left[\mathrm{Fe}(\mathrm{CO})_{5}\right],\left[\mathrm{Os}(\mathrm{CO})_{5}\right],\left[\mathrm{Rh}(\mathrm{CO})_{4.5}\right]$, and $\left[\mathrm{Pd}(\mathrm{CO})_{4}\right]$ can be utilized to generate other higher respective clusters. A scheme for generating cluster formulas is shown in SC-1.

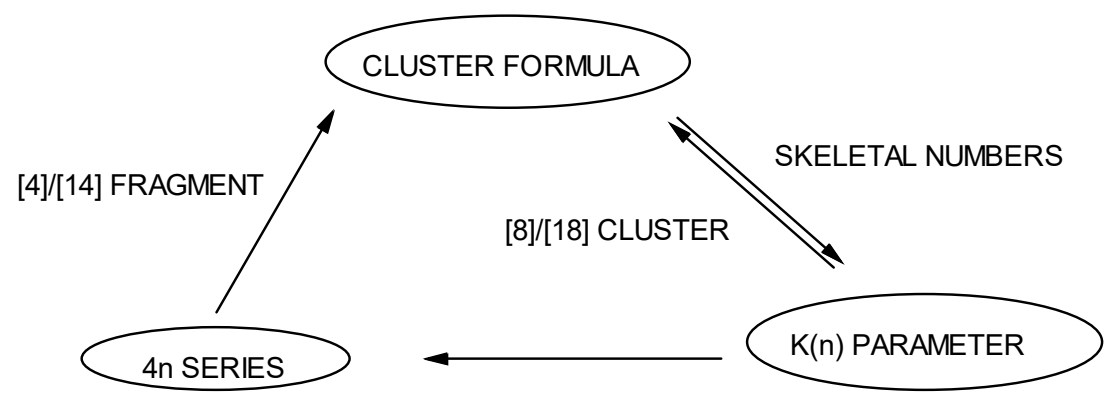

SC-1

\section{Results and Discussions}

The Primary Cluster and multiples of K(n) parameter. In earlier work, transition elements, main group elements and ligands were assigned skeletal numbers (Kiremire, 2016d). Let us take iron, Fe as a skeletal element to define a primary cluster based on series method. The element has a skeletal number of $5,[\mathrm{Fe}(\mathrm{K}=5)]$. This was interpreted to mean that 
Fe skeletal element requires 5 electron pairs or 5 two electron donor ligands in order for it to obey the 18 electron rule. If we take a $\mathrm{CO}$ as representative of a 2 -electron donor ligand, we can express this as $\mathrm{Fe}+5 \mathrm{CO} \rightarrow \mathrm{Fe}(\mathrm{CO})_{5}$. The cluster, $\mathrm{Fe}(\mathrm{CO})_{5}$, can be referred to as a PRIMARY CLUSTER for all Fe carbonyl clusters. The other multi-skeletal cluster of Fe may simply be regarded as suitable multiples of the primary cluster, $\mathrm{Fe}(\mathrm{CO})_{5}$. For instance, $\mathrm{Fe}_{2}(\mathrm{CO})_{9}$, may hypothetically be regarded as a derivative of $\mathrm{Fe}(\mathrm{CO})_{5}$ by the following simple re-arrangement;

$2[\mathrm{Fe}(\mathrm{CO}) 5]-1(\mathrm{CO}) \rightarrow \mathrm{Fe}_{2}(\mathrm{CO})_{9}[\mathrm{~K}=1]$. This can also be expressed in another way;

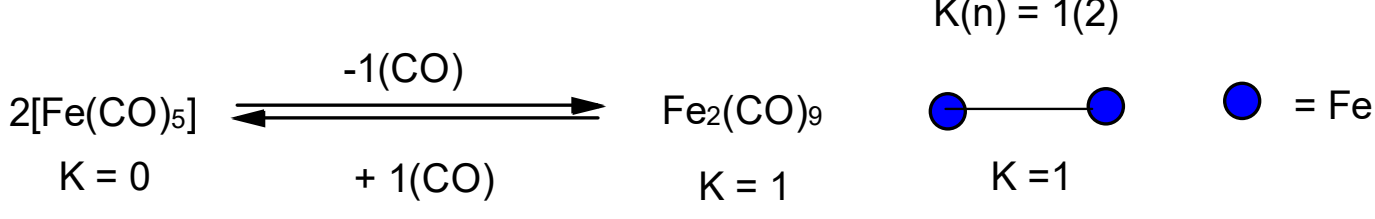

Equation 1.

The complex $\mathrm{Fe}(\mathrm{CO})_{5}$ has a $\mathrm{K}$ value of zero. According to the hypothetical equation 1, by combining $2 \mathrm{Fe}(\mathrm{CO})_{5}$ primary clusters and removing $1(\mathrm{CO})$ ligand, we create a dimeric type of complex, $\mathrm{Fe}_{2}(\mathrm{CO})_{9}$ which has a skeletal linkage $\mathrm{K}=1$, that is, one $\mathrm{Fe}-\mathrm{Fe}$ metal-metal bond has been formed by the forward hypothetical reaction of equation 1 . In previous work, it was explained that $\mathrm{K}(\mathrm{n})$ parameter has a double meaning, that is, the number of linkages binding the skeletal elements together or the shortage number of electron pairs needed by a cluster to split up into primary clusters all of which obey 18 electron or 8 electron rule(Kiremire, 2017c). The reverse reaction of equation 1 means that since $\mathrm{Fe}_{2}(\mathrm{CO})_{9}$ cluster has a $\mathrm{K}=1$ value, it requires one 2-elctron donor for it to decompose into 2 primary clusters. Thus, $\mathrm{Fe}_{2}(\mathrm{CO})_{9}+1 \mathrm{CO} \rightarrow 2\left[\mathrm{Fe}(\mathrm{CO})_{5}\right]$. Thus, equation 1 resembles an equilibrium type of a reaction.

The PRIMARY CLUSTERS of transition metal skeletal elements can be expressed as $\mathrm{Sc}\left(\mathrm{K}=7.5, \mathrm{Sc}(\mathrm{CO})_{7.5}\right]$; $\operatorname{Ti}(\mathrm{K}=7)$, $\mathrm{Ti}(\mathrm{CO})_{7} ; \mathrm{V}\left(\mathrm{K}=6.5, \mathrm{~V}(\mathrm{CO})_{6.5} ; \mathrm{Cr}(\mathrm{K}=6), \mathrm{Cr}(\mathrm{CO})_{6} ; \mathrm{Mn}(\mathrm{K}=5.5), \mathrm{Mn}(\mathrm{CO}) 5.5 ; \mathrm{Fe}(\mathrm{K}=5), \mathrm{Fe}(\mathrm{CO})_{5} ; \mathrm{Co}(\mathrm{K}=4.5), \mathrm{Co}(\mathrm{CO})_{4.5}\right.$; $\mathrm{Ni}(\mathrm{K}=4), \mathrm{Ni}(\mathrm{CO})_{4} ; \mathrm{Cu}(\mathrm{K}=3.5), \mathrm{Cu}(\mathrm{CO})_{3.5} ; \mathrm{Zn}(\mathrm{K}=3), \mathrm{Zn}(\mathrm{CO})_{3}$.

Examples are given in schemes SC-1, SC-2, SC-3 and sc-4.

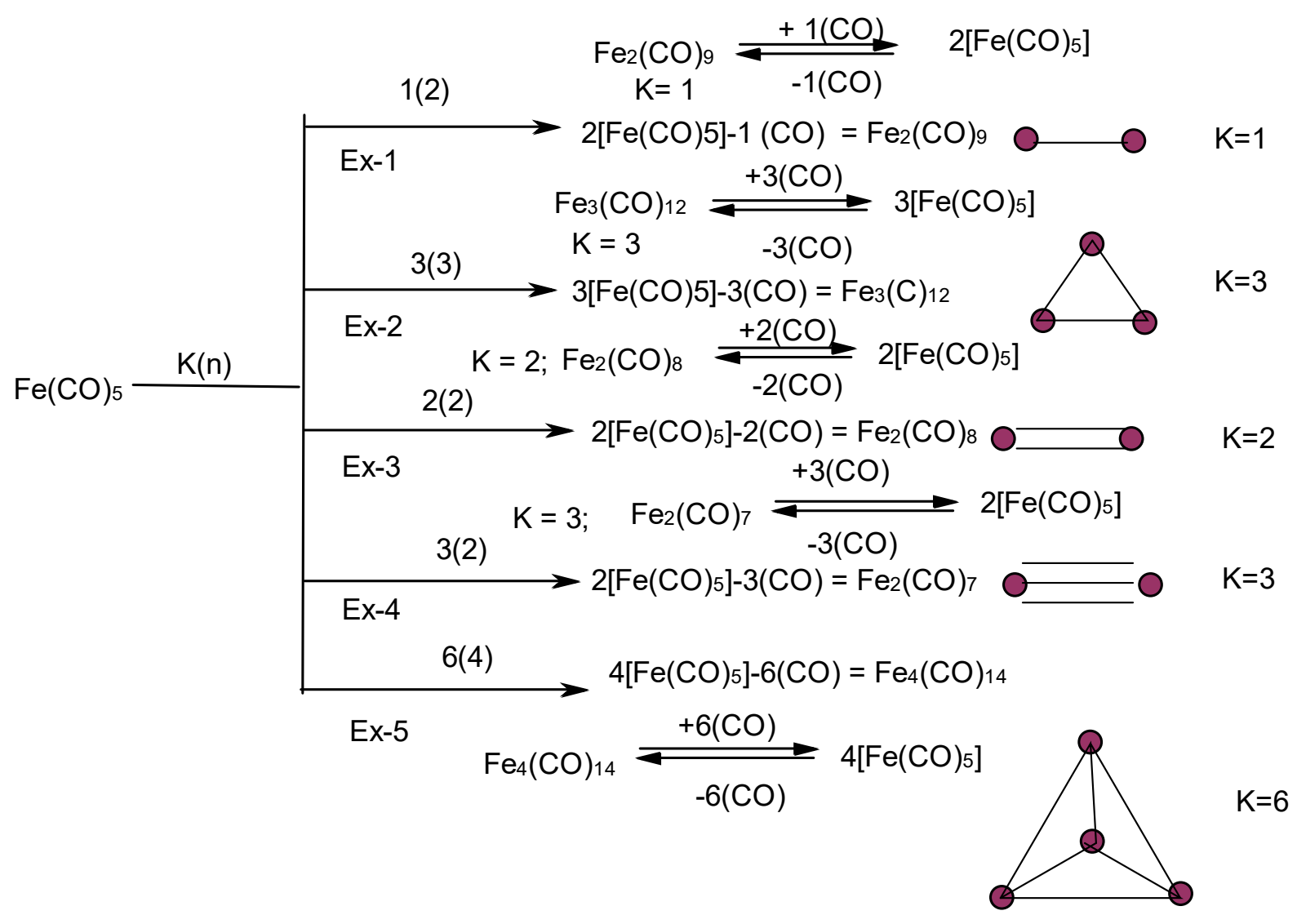

SC-2 


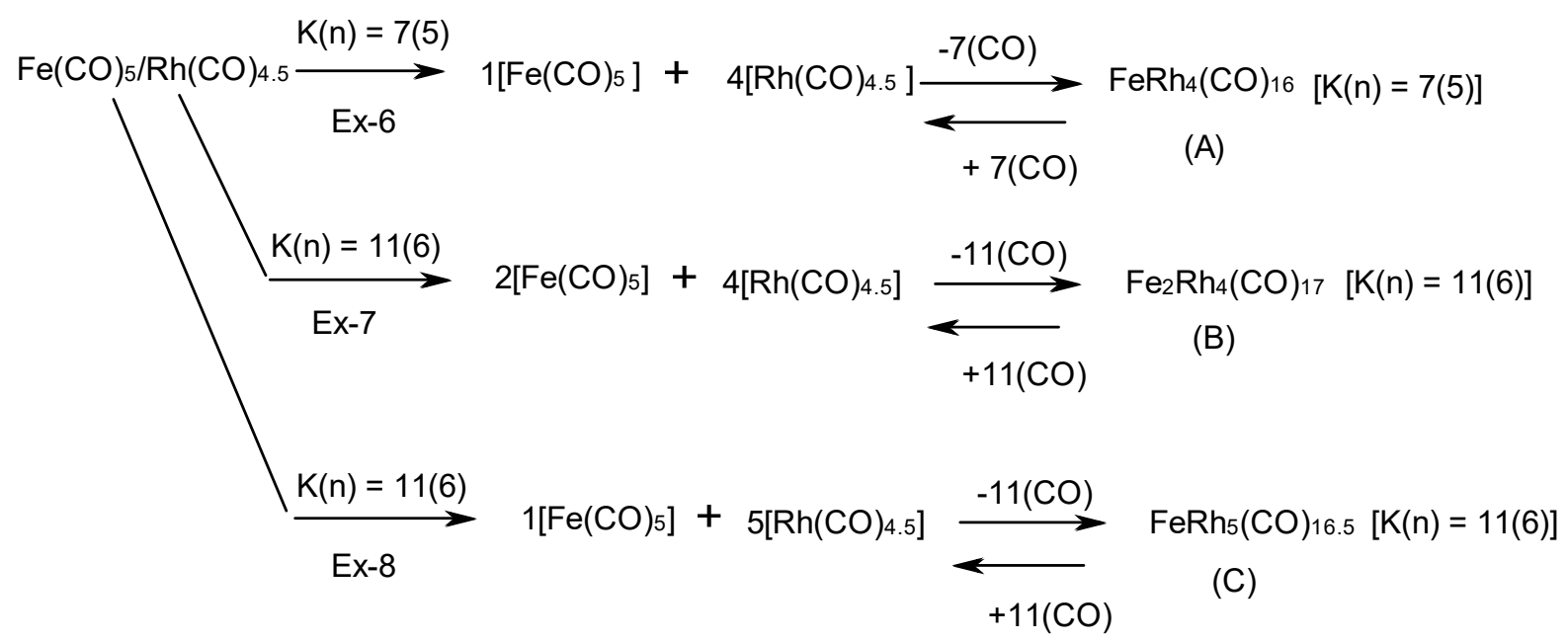

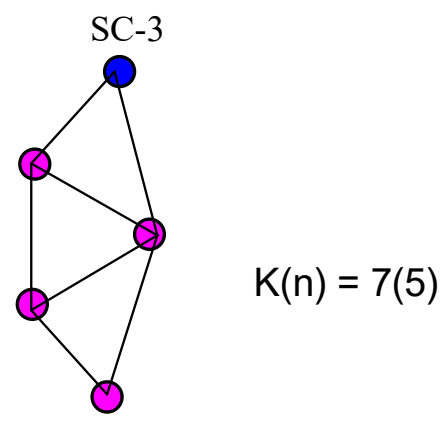

Possible sketch of skeletal isomer of $(A)$

F-1

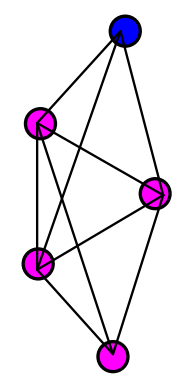

Reported skeletal structure (A)

$\mathrm{F}-2$

The possible skeletal shapes of clusters shown in scheme SC-3 are sketched in F-1, F-2, F-3, F-4, and F-5. An example of determining possible distribution of ligands is shown in for F-5 is sketched in F-6. The short-cut method of deriving the type of series from $\mathrm{K}(\mathrm{n})$ is shown in F-7. 
$\mathrm{Fe}_{2} \mathrm{Rh}_{4}(\mathrm{CO}){ }_{17}$

$K(n)=11(6)$

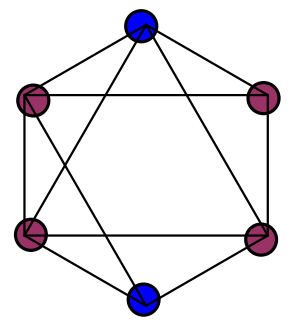

F-3
(B)

$$
K(n)=11(6)
$$

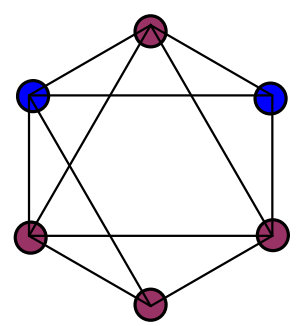

F-4

Possible skeletal isomers of (B)

$\mathrm{FeRh}_{5}(\mathrm{CO}) 16.5$

(C)

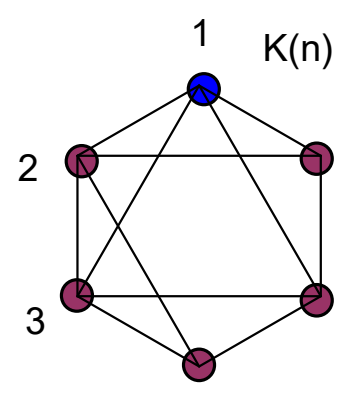

4

Possible skeletal isomers of (C)

$\mathrm{K} 1=5-2=3$

$\mathrm{K} 2=4.5-2=2.5$

$\mathrm{K} 3=\mathrm{K} 2=2.5$

$\mathrm{K} 4=4.5-1.5=3$

$\mathrm{K} 5=\mathrm{K} 2=2.5$

5

$\mathrm{K} 6=\mathrm{K} 4=3$

TOTAL $=16.5$

\section{F-5}

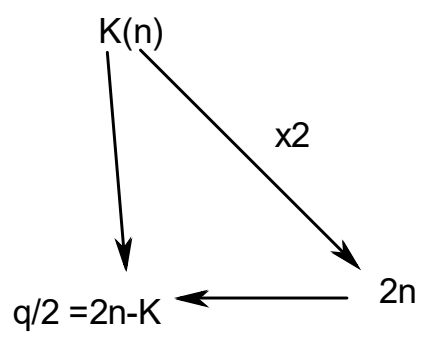

$K(n)=7(5) ; q / 2=10-7=3, q=6, S=4 n+6$, ARACHNO SERIES $\mathrm{Kp}=\mathrm{C}^{-2} \mathrm{C}[\mathrm{M} 7]$ THE CLUSTER BELONGS TO CLUSTER

GROUP 7 BUT 2 LEVELS BELOW THE CLOSO BASELINE.

$\mathrm{K}(\mathrm{n})=11(6), \mathrm{S}=4 \mathrm{n}+2$, CLOSO SERIES. $\mathrm{Kp}=\mathrm{C}^{0} \mathrm{C}[\mathrm{M} 6]$

THE CLUSTER BELONGS TO THE CLUSTER GROUP 6.

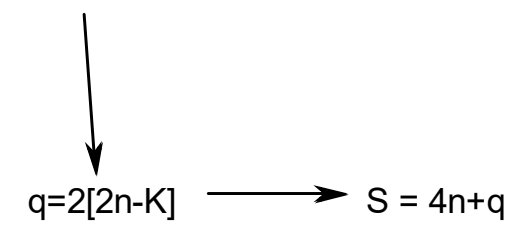

SHORT-CUT FOR CATEGORIZATION OF CLUSTERS.

f-7 
- $\mathrm{Fe}_{6}(\mathrm{C})(\mathrm{CO}){ }_{16}^{2-} \mathrm{K}=6[5]-2-16-1=11, \mathrm{~K}(\mathrm{n})=11(6), \mathrm{S}=4 \mathrm{n}+2$

Ex-9

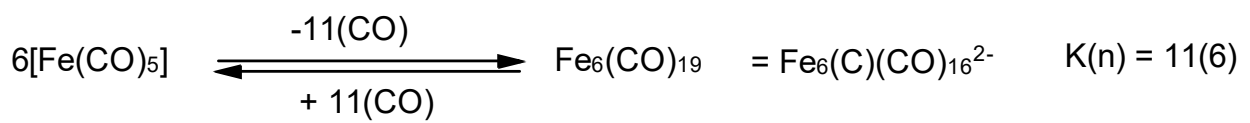

$\mathrm{Fe}_{4} \mathrm{Au}_{5}(\mathrm{CO}){ }_{16}^{3-:} \mathrm{K}=4[5]+5[3.5]-16-1.5=20, \mathrm{~K}(\mathrm{n})=20(9), \mathrm{S}=4 \mathrm{n}-4, \mathrm{Kp}=\mathrm{C}^{3} \mathrm{C}[\mathrm{M} 6]$

Ex-10

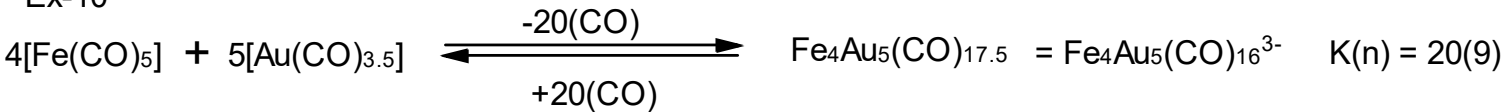

$$
+20(\mathrm{CO})
$$

- $\mathrm{Fe}_{4} \mathrm{Rh}_{2}(\mathrm{C})(\mathrm{CO})_{15}(\mathrm{AuL}) 2: \mathrm{K}=4[5]+2[4.5]+2[3.5-1]-2-15=17(8) ; \mathrm{K}(\mathrm{n})=\mathrm{S}=4 \mathrm{n}-2, \mathrm{Kp}=\mathrm{C}^{2} \mathrm{C}[\mathrm{M} 6]$

Ex-11

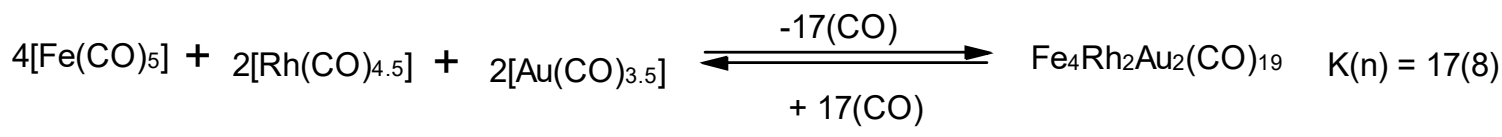

\section{SC-4}

\subsection{Osmium Carbonyl Clusters}

The derivation of selected osmium carbonyl clusters using the primary cluster and $\mathrm{K}(\mathrm{n})$ parameter is summarized in scheme SC-5. Since the cluster number (K value) of osmium is a whole number $(K=5)$, the multiples of $K(n)$ and the primary cluster, $\mathrm{Os}(\mathrm{CO})_{5}$ come out as with carbonyl ligands as whole numbers regardless as to whether the cluster has a charge on it or not. On the other hand, a skeletal element such as rhodium which has a fractional $\mathrm{K}$ value $[\mathrm{Rh}(\mathrm{K}=4.5)]$, the cluster will have the number of ligands with a whole number if the nuclearity is even. Otherwise, the number of ligands will have a fractional value. Since osmium has a $\mathrm{K}$ value whole number, the effective number of ligands to combine with osmium skeletal elements will be a whole number. This is shown in Table 1. Since osmium and palladium skeletal elements have $\mathrm{K}$ values of whole numbers, their skeletal elements will combine with whole number ligands. On the other hand, the transition metal skeletal elements with fractional $\mathrm{K}$ values will combine with effective whole number ligands if their nuclearity indices are even, but if odd, then the effective number of ligands will be odd number. This demonstrated in Table 2. 


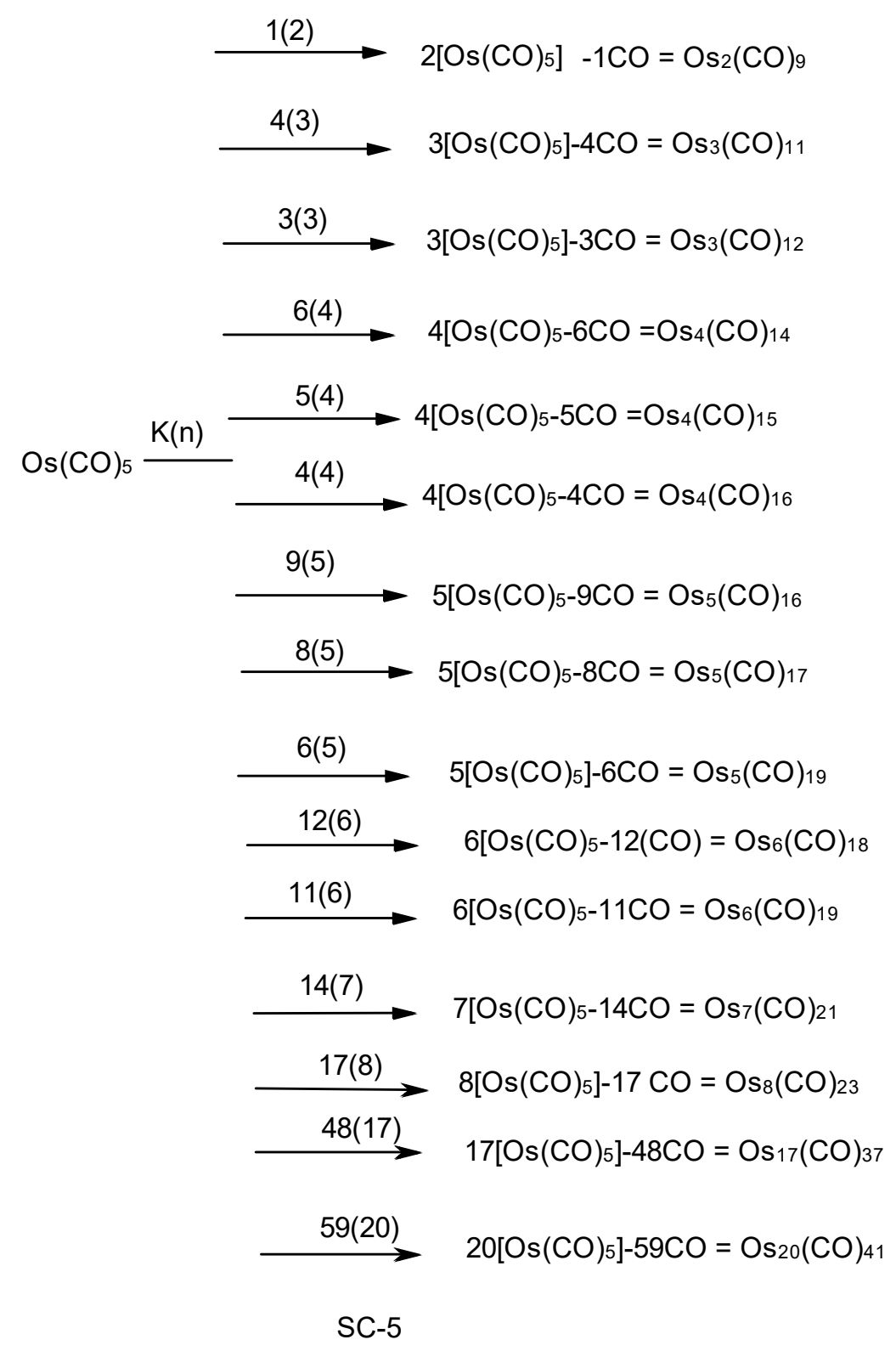




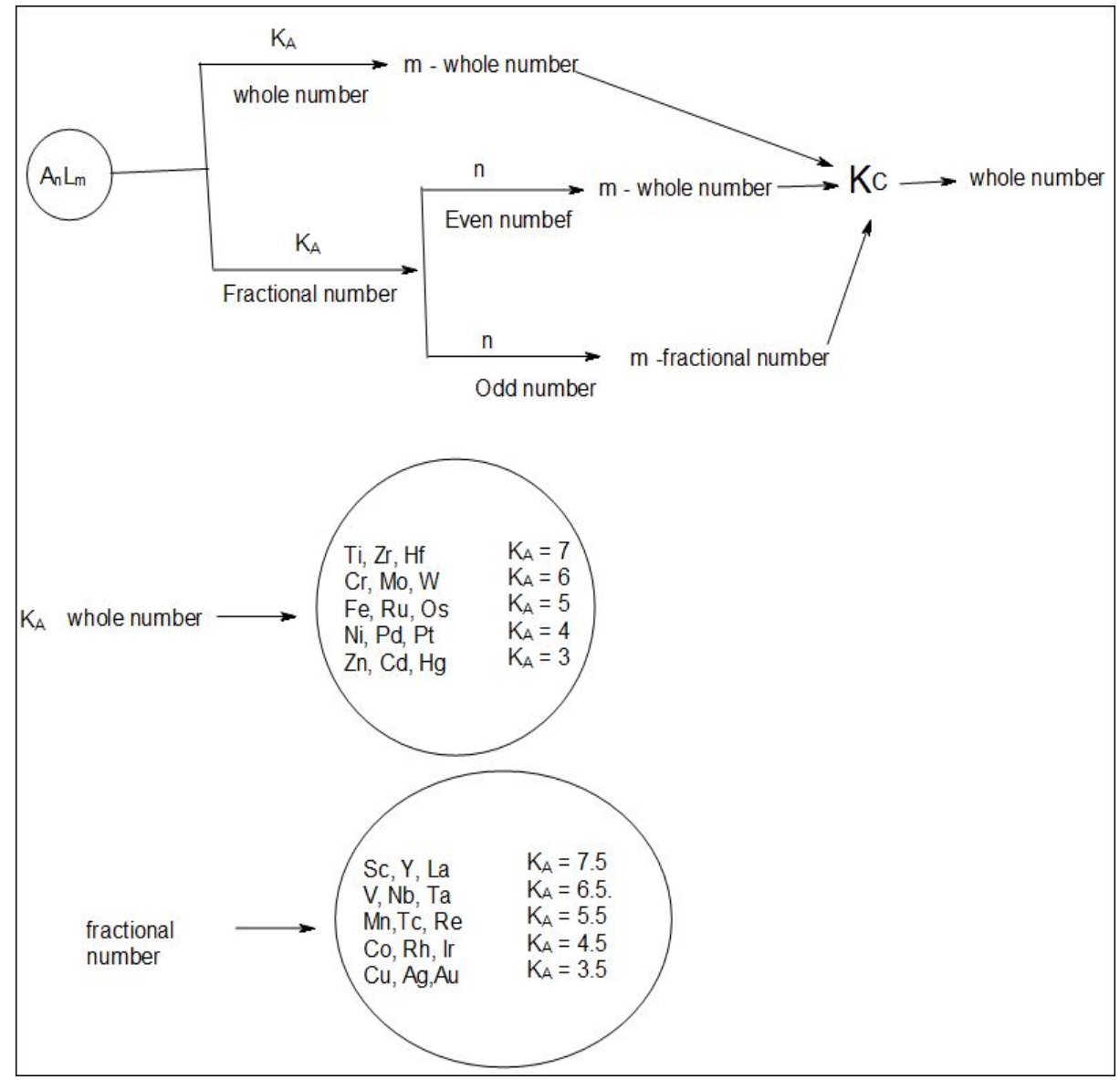

$\mathrm{A}_{\mathrm{n}} \mathrm{L}_{\mathrm{m}}$ - cluster, $\mathrm{K}_{\mathrm{C}}$ - cluster number, $\mathrm{K}_{\mathrm{A}}$ - cluster number of skeletal element, $\mathrm{A}$ - skeletal element, $\mathrm{L}$ - ligand SC-6

Table 1. Selected Osmium and Palladium Clusters

\begin{tabular}{|c|c|c|c|c|c|}
\hline Equivalents & Os $(\mathrm{K}=5)$ & $\mathrm{Kc}$ & Equivalents & $\operatorname{Pd}(\mathrm{K}=4)$ & $\mathrm{Kc}$ \\
\hline & $\mathrm{Os}(\mathrm{CO})_{5}$ & $1[5]-5(1)=0$ & & $\mathrm{Pd}(\mathrm{CO})_{4}$ & $4-4=0$ \\
\hline & $\mathrm{Os}_{2}(\mathrm{CO})_{9}$ & $2[5]-9=1$ & $\mathrm{Pd}_{3}(\mathrm{CO})_{3} \mathrm{~L}_{3}$ & $\mathrm{Pd}_{3}(\mathrm{CO})_{6}$ & $12-6=6$ \\
\hline & $\mathrm{Os}_{3}(\mathrm{CO})_{12}$ & $15-12=3$ & $\mathrm{Pd}_{10}(\mathrm{CO})_{12} \mathrm{~L}_{6}$ & $\mathrm{Pd}_{10}(\mathrm{CO})_{18}$ & $40-18=22$ \\
\hline $\mathrm{Os}_{3} \mathrm{H}(\mathrm{CO})_{11^{-}}$ & $\mathrm{Os}_{3}(\mathrm{CO})_{12}$ & $15-12=3$ & $\mathrm{Pd}_{12}(\mathrm{CO})_{15} \mathrm{~L}_{3}$ & $\mathrm{Pd}_{12}(\mathrm{CO})_{18}$ & $48-18=30$ \\
\hline \multirow[t]{2}{*}{$\mathrm{Os}_{3} \mathrm{H}_{2}(\mathrm{CO})_{11}$} & $\mathrm{Os}_{3}(\mathrm{CO})_{12}$ & $15-12=3$ & $\mathrm{Pd}_{12}(\mathrm{CO})_{15} \mathrm{~L}_{7}$ & $\mathrm{Pd}_{12}(\mathrm{CO})_{22}$ & $48-22=26$ \\
\hline & $\mathrm{Os}_{4}(\mathrm{CO})_{14}$ & $20-14=6$ & $\mathrm{Pd}_{16}(\mathrm{CO})_{7} \mathrm{~L}_{6}$ & $\mathrm{Pd}_{16}(\mathrm{CO})_{13}$ & $64-13=51$ \\
\hline $\mathrm{Os}_{4} \mathrm{H}_{2}(\mathrm{CO})_{12}{ }^{2-}$ & $\mathrm{Os}_{4}(\mathrm{CO})_{14}$ & $20-14=6$ & $\mathrm{Pd}_{23}(\mathrm{CO})_{22} \mathrm{~L}_{10}$ & $\mathrm{Pd}_{23}(\mathrm{CO})_{32}$ & $92-32=60$ \\
\hline $\mathrm{Os}_{4} \mathrm{H}_{2}(\mathrm{CO})_{12}{ }^{2}$ & $\mathrm{Os}_{4}(\mathrm{CO})_{14}$ & $20-14=6$ & $\mathrm{Pd}_{23}(\mathrm{CO})_{20} \mathrm{~L}_{10}$ & $\mathrm{Pd}_{23}(\mathrm{CO})_{30}$ & $92-30=62$ \\
\hline $\mathrm{Os}_{4} \mathrm{H}_{3}(\mathrm{CO})_{12^{-}}^{-}$ & $\mathrm{Os}_{4}(\mathrm{CO})_{14}$ & $20-14=6$ & $\mathrm{Pd}_{30}(\mathrm{CO})_{26} \mathrm{~L}_{10}$ & $\mathrm{Pd}_{30}(\mathrm{CO})_{36}$ & $120-36=84$ \\
\hline \multirow{2}{*}{$\mathrm{Os}_{4} \mathrm{H}_{4}(\mathrm{CO})_{12}$} & $\mathrm{Os}_{4}(\mathrm{CO})_{14}$ & $20-14=6$ & $\mathrm{Pd}_{29} \mathrm{Ni}_{3}(\mathrm{CO})_{22} \mathrm{~L}_{3}$ & $\mathrm{Pd}_{32}(\mathrm{CO})_{25}$ & $128-25=103$ \\
\hline & $\mathrm{Os}_{5}(\mathrm{CO})_{16}$ & $25-16=9$ & $\mathrm{Pd}_{34}(\mathrm{CO})_{24} \mathrm{~L}_{12}$ & $\mathrm{Pd}_{34}(\mathrm{CO})_{36}$ & $136-36=100$ \\
\hline \multirow[t]{2}{*}{$\mathrm{Os}_{5} \mathrm{H}(\mathrm{CO})_{15^{-}}$} & $\mathrm{Os}_{5}(\mathrm{CO})_{16}$ & $25-16=9$ & $\mathrm{Pd}_{36}(\mathrm{CO})_{28} \mathrm{~L}_{14}$ & $\mathrm{Pd}_{36}(\mathrm{CO})_{42}$ & $144-42=102$ \\
\hline & $\mathrm{Os}_{6}(\mathrm{CO})_{18}$ & $30-18=12$ & $\mathrm{Pd}_{38}(\mathrm{CO})_{28} \mathrm{~L}_{12}$ & $\mathrm{Pd}_{38}(\mathrm{CO})_{40}$ & $152-40=112$ \\
\hline $\mathrm{Os}_{6}(\mathrm{CO})_{18}{ }^{2-}$ & $\mathrm{Os}_{6}(\mathrm{CO})_{19}$ & $30-19=11$ & $\mathrm{Pd}_{20} \mathrm{Ni}_{26}(\mathrm{CO})_{54}{ }^{6}$ & $\mathrm{Pd}_{46}(\mathrm{CO})_{57}$ & $184-57=127$ \\
\hline $\mathrm{Os}_{6} \mathrm{H}(\mathrm{CO})_{18^{-}}$ & $\mathrm{Os}_{6}(\mathrm{CO})_{19}$ & $30-19=11$ & $\mathrm{Pd}_{52}(\mathrm{CO})_{36} \mathrm{~L}_{14}$ & $\mathrm{Pd}_{52}(\mathrm{CO})_{50}$ & $208-50=158$ \\
\hline \multirow[t]{2}{*}{$\mathrm{Os}_{6} \mathrm{H}_{2}(\mathrm{CO})_{18}$} & $\mathrm{Os}_{6}(\mathrm{CO})_{19}$ & $30-19=11$ & $\mathrm{Pd}_{54}(\mathrm{CO})_{40} \mathrm{~L}_{14}$ & $\mathrm{Pd}_{54}(\mathrm{CO})_{54}$ & $216-54=162$ \\
\hline & $\mathrm{Os}_{7}(\mathrm{CO})_{21}$ & $35-21=14$ & & & \\
\hline $\mathrm{Os}_{7} \mathrm{H}_{2}(\mathrm{CO})_{20}$ & $\mathrm{Os}_{7}(\mathrm{CO})_{21}$ & $35-21=14$ & & & \\
\hline $\mathrm{Os}_{8}(\mathrm{CO})_{22}{ }^{2-}$ & $\mathrm{Os}_{8}(\mathrm{CO})_{23}$ & $40-23=17$ & & & \\
\hline $\mathrm{Os}_{8} \mathrm{H}(\mathrm{CO})_{22}{ }^{-}$ & $\mathrm{Os}_{8}(\mathrm{CO})_{23}$ & $40-23=17$ & & & \\
\hline $\mathrm{Os}_{9}(\mathrm{CO})_{24}{ }^{2-}$ & $\mathrm{Os}_{9}(\mathrm{CO})_{25}$ & $45-25=20$ & & & \\
\hline $\mathrm{Os}_{9} \mathrm{H}(\mathrm{CO})_{24^{-}}$ & $\mathrm{Os}_{9}(\mathrm{CO})_{25}$ & $45-25=20$ & & & \\
\hline $\mathrm{Os}_{10}(\mathrm{CO})_{26}{ }^{2-}$ & $\mathrm{Os}_{10}(\mathrm{CO})_{27}$ & $50-27=23$ & & & \\
\hline $\mathrm{Os}_{20}(\mathrm{CO})_{40}{ }^{2}$ & $\mathrm{Os}_{20}(\mathrm{CO})_{41}$ & $100-41=59$ & & & \\
\hline
\end{tabular}


Let us consider the case of rhodium clusters. The $\mathrm{K}$ value of rhodium is 4.5. For 2 rhodium skeletal elements, $\mathrm{K}=2[4.5]$ $=9$. This is a whole number and the 2 skeletal elements must combine with a whole number of ligands in order to produce a whole number $\mathrm{K}$ value and hence, we can have $\mathrm{Rh}_{2}(\mathrm{CO})_{8}(\mathrm{~K}=1)$. Similarly, $\mathrm{Rh}_{4}(\mathrm{~K}=18)$ can have a cluster $\mathrm{Rh}_{4}(\mathrm{CO})_{12}(\mathrm{~K}=18-12=6)$ while $\mathrm{Rh}_{6}(\mathrm{~K}=27)$ can have $\mathrm{Rh}_{6}(\mathrm{CO})_{16}(\mathrm{~K}=27-16=11)$. Other clusters in this category are $\mathrm{Rh}_{12}(\mathrm{CO})_{29}, \mathrm{Rh}_{10}(\mathrm{CO})_{22}$, and $\mathrm{Rh}_{22}(\mathrm{CO})_{39}$. On the other hand, $\mathrm{Rh}_{7}(\mathrm{~K}=7 \mathrm{x} 4.5=31.5)$ can combine with fractional $\mathrm{CO}$ ligands to form the cluster $\mathrm{Rh}_{7}(\mathrm{CO})_{17.5}(\mathrm{~K}=31.5-17.5=14)$. Other clusters in this category include, $\mathrm{Rh}_{9}(\mathrm{CO})_{20.5}(\mathrm{~K}=$ $40.5-20.5=20)$, and $\mathrm{Rh}_{11}(\mathrm{CO})_{24.5}(\mathrm{~K}=49.5-24.5=25)$. In view of this, it is surprising that clusters such as $\mathrm{Au}_{8} \mathrm{~L}_{8}{ }^{2+}, \mathrm{Au}_{8}$, $\mathrm{K}=28, \mathrm{~K}=28-8+1=21, \mathrm{~K}(\mathrm{n})=21(8) ; \mathrm{Au}_{8} \mathrm{~L}_{7}{ }^{2+}, \mathrm{Au}_{8}, \mathrm{~K}=28-7+1=22, \mathrm{~K}(\mathrm{n})=22(8) ; \mathrm{Au}_{13} \mathrm{~L}_{10} \mathrm{Cl}_{2}{ }^{3+}, \mathrm{Au}_{13}, \mathrm{~K}=45.5, \mathrm{~K}_{\mathrm{C}}=$ $45.5-10-1+1.5=36, \mathrm{~K}(\mathrm{n})=36(13) ; \mathrm{Au}_{25} \mathrm{~L}_{10} \mathrm{Cl}_{2} \mathrm{R}_{5}{ }^{2+}, \mathrm{Au} 25, \mathrm{~K}=87.5, \mathrm{~K}_{\mathrm{C}}=87.5-10-1-2.5+1=75,-\mathrm{K}(\mathrm{n})=75(25)$ and $\mathrm{Au}_{38} \mathrm{~L}_{18} \mathrm{Cl}_{2}{ }^{4+}$, $\mathrm{Au} 38, \mathrm{~K}=133, \mathrm{KC}=133-18-1+2=116, \mathrm{~K}(\mathrm{n})=116(38)$; should have positive and whole number $\mathrm{K}(\mathrm{n})$ values. It is therefore surprising that $\mathrm{Au}_{55} \mathrm{~L}_{12} \mathrm{Cl}_{6} \mathrm{Au}_{55}, \mathrm{~K}=192.5, \mathrm{KC}=192.5-12-3=177.5$ has got a fractional $\mathrm{K}(\mathrm{n})=$ 177.5(55) value (Coenraad, 2006; Gimeno, 2008; Konishi, 2014 ; Puls, 2014)

\subsection{Selected K(n) Cluster Groups}

In previous work, it was discovered that the main group and transition metals are interrelated through the following isolobal equilibrium relationship of the series.

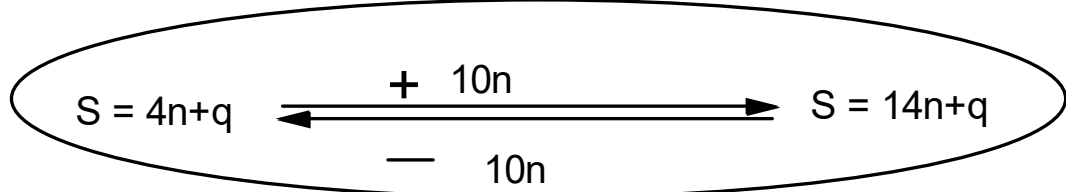

Since clusters of the main group and transition metals are interrelated, it was proposed that they be categorized into cluster groups (Kiremire, 2018b). Furthermore, the closo baseline $(S=4 n+2)$ was selected to be used as a reference point. If we use the capping symbol for the closo baseline as $\mathrm{Kp}=\mathrm{C}^{0} \mathrm{C}[\mathrm{Mx}]$, then the cluster groups could be based upon the numerical value of $x$. If we take boranes as examples of baseline clusters, then [M1], $S=4 n+2, K=2 n-1=$ $2(1)-1=1$ and $\mathrm{K}(\mathrm{n})=1(1)$ and the borane cluster will be $[\mathrm{BH}](1)+2 \mathrm{H}=\mathrm{BH}_{3}\left[\mathrm{BH}^{2-}\right]$. The $[\mathrm{M} 2]$ will have the $\mathrm{K}(\mathrm{n})=$ $3(2)$, and $\mathrm{F}_{\mathrm{B}}=[\mathrm{BH}](2)+2 \mathrm{e}=\mathrm{B}_{2} \mathrm{H}_{2}{ }^{2-}$; [M3], $\mathrm{K}(\mathrm{n})=5(3), \mathrm{F}_{\mathrm{B}}=\mathrm{B}_{3} \mathrm{H}_{3}{ }^{2-}$; [M4], $\mathrm{K}(\mathrm{n})=7(4), \mathrm{F}_{\mathrm{B}}=\mathrm{B}_{4} \mathrm{H}_{4}{ }^{2-}$; [M5], $\mathrm{K}(\mathrm{n})=$ 9(5), $\mathrm{F}_{\mathrm{B}}=\mathrm{B}_{5} \mathrm{H}_{5}{ }^{2-} ;[\mathrm{M} 6], \mathrm{K}(\mathrm{n})=11(6), \mathrm{F}_{\mathrm{B}}=\mathrm{B}_{6} \mathrm{H}_{6}{ }^{2-} ;[\mathrm{M} 7], \mathrm{K}(\mathrm{n})=13(7), \mathrm{F}_{\mathrm{B}}=\mathrm{B}_{7} \mathrm{H}_{7}{ }^{2-} ;[\mathrm{M} 8], \mathrm{K}(\mathrm{n})=15(8), \mathrm{F}_{\mathrm{B}}=$ $\mathrm{B}_{8} \mathrm{H}_{8}{ }^{2-} ;[\mathrm{M} 9], \mathrm{K}(\mathrm{n})=17(9), \mathrm{F}_{\mathrm{B}}=\mathrm{B}_{9} \mathrm{H}_{9}{ }^{2-} ;[\mathrm{M} 10], \mathrm{K}(\mathrm{n})=19(10), \mathrm{F}_{\mathrm{B}}=\mathrm{B}_{10} \mathrm{H}_{10}{ }^{2-} ;[\mathrm{M} 11], \mathrm{K}(\mathrm{n})=21(11), \mathrm{F}_{\mathrm{B}}=\mathrm{B}_{11} \mathrm{H}_{11}{ }^{2-}$ and [M12], $\mathrm{K}(\mathrm{n})=23(12), \mathrm{F}_{\mathrm{B}}=\mathrm{B}_{12} \mathrm{H}_{12}{ }^{2-}$. The $\mathrm{K}(\mathrm{n})$ numerical series selected for the golden clusters cover the range [Mx], $\mathrm{x}=-6$ to $\mathrm{x}=+6$. These series are given in Table 1. The table is general for all clusters from the main group or transition metals. The selected portion of Table 1 represents all the chemical clusters capping under that domain. The $\mathrm{S}=4 \mathrm{n}+2$ represents zero capping $\mathrm{Kp}=\mathrm{C}^{0}, 4 \mathrm{n}+0 \rightarrow$ mono-capping, $\mathrm{Kp}=\mathrm{C}^{1} ; 4 \mathrm{n}-2 \rightarrow$ bi-capping, $\mathrm{Kp}=\mathrm{C}^{2} ; 4 \mathrm{n}-4, \mathrm{Kp}=\mathrm{C}^{3} ; 4 \mathrm{n}-6, \mathrm{Kp}$ $=C^{4} ; 4 n-8, K p=C^{5}$; and $4 n-10, K p=C^{6}$ and so on. The corresponding numerical capping $K(n)$ series for octahedral-based [M6] clusters are 11(6), 14(7), 17(8), 20(9), 23(10), 26(11) and 29(12) respectively. Other selected numerical $\mathrm{K}(\mathrm{n})$ series cover the range $[\mathrm{Mx}], \mathrm{x}=-6$ to +6 are given in Table 3 .

\subsection{Golden Cluster Series}

The use of K(n) parameter and the Primary Cluster of 18 electron valence content of osmium $\mathrm{Os}(\mathrm{CO})_{5}$ to generate the required osmium clusters was illustrated in $\mathrm{SC}-2$ in which the skeletal number for osmium $\mathrm{K}=5$. The procedure was also summarized in SC-3. This procedure was arrived at after a careful scrutiny of the relationship between a given simple cluster formula and its K(n) parameter. The will be interested to know that in fact the procedure came from knowing that the cluster $\mathrm{Fe}_{2}(\mathrm{CO})_{9}$, has one single skeletal bond $(\mathrm{K}=1)$ that links two skeletal elements $(\mathrm{n}=2)$, hence $\mathrm{K}(\mathrm{n})=1(2)$ and that the 18 electron mono-skeletal cluster of iron has a formula $\mathrm{Fe}(\mathrm{CO})_{5}$ in which $\mathrm{Fe}(\mathrm{K}=5)$. After a deeper thought, it became clear that we could regard $\mathrm{Fe}_{2}(\mathrm{CO})_{9} \equiv 2\left[\mathrm{Fe}(\mathrm{CO})_{5}\right]-1 \mathrm{CO}$ which resembles $(\mathrm{n}=2)\left[\mathrm{Fe}(\mathrm{CO})_{5}\right]-(\mathrm{K}=1)(\mathrm{CO})$. The coefficients are simply the reverse of $\mathrm{K}(\mathrm{n})=1(2)$. Applying the same approach for $\mathrm{K}(\mathrm{n})$ $=3(3)$ on $\mathrm{Fe}(\mathrm{CO})_{5}$, we get $3\left[\mathrm{Fe}(\mathrm{CO})_{5}\right]-3 \mathrm{CO}=\mathrm{Fe}_{3}(\mathrm{CO})_{12}$ a well known cluster, and $\mathrm{K}(\mathrm{n})=9(5)$, gives us $5[\mathrm{Fe}(\mathrm{CO}) 5]-9 \mathrm{CO}=\mathrm{Fe}_{5}(\mathrm{CO})_{16}$ an analogue of $\mathrm{Os}_{5}(\mathrm{CO})_{16}$ another cluster with a trigonal bipyramid skeletal shape(Hughes and Wade, 2000). In the case of gold, the mono-skeletal element, [Au] with 11 valence electrons, it will require 3.5 two electron donor ligands to make a primary cluster $\left[\mathrm{AuL}_{3.5}\right]$ of 18 valence electron content cluster. By following the same procedure as illustrated in SC-2 and summarized in SC-3, using the $\mathrm{K}(\mathrm{n})$ values in Table 1A and 1B and the primary cluster $\left[\mathrm{AuL}_{3.5}\right]$, the hypothetical golden clusters shown in Tables $2 \mathrm{~A}$ and $2 \mathrm{~B}$ were obtained.

Let us take a few more examples to illustrate this point by deriving the golden clusters corresponding to the K(n) values highlighted blue in Table 3. 
- $\mathrm{K}(\mathrm{n})=20(7): \quad \mathrm{F}_{\mathrm{G} 1}=7\left[\mathrm{AuL}_{3.5}\right]-20 \mathrm{~L}=\mathrm{Au}_{7} \mathrm{~L}_{4.5} ; \mathrm{S}=4 \mathrm{n}-12, \mathrm{Kp}=\mathrm{C}^{7} \mathrm{C}[\mathrm{M} 0]$

- $\mathrm{K}(\mathrm{n})=22(8): \mathrm{F}_{\mathrm{G} 2}=8\left[\mathrm{AuL}_{3.5}\right]-22 \mathrm{~L}=\mathrm{Au}_{8} \mathrm{~L}_{6} ; \mathrm{S}=4 \mathrm{n}-12, \mathrm{Kp}=\mathrm{C}^{7} \mathrm{C}[\mathrm{M} 1]$

- $\mathrm{K}(\mathrm{n})=24(9): \quad \mathrm{F}_{\mathrm{G} 3}=9\left[\mathrm{AuL}_{3.5}\right]-24 \mathrm{~L}=\mathrm{Au}_{9} \mathrm{~L}_{7.5} ; \mathrm{S}=4 \mathrm{n}-12, \mathrm{Kp}=\mathrm{C}^{7} \mathrm{C}[\mathrm{M} 2]$

- $\mathrm{K}(\mathrm{n})=26(10): \mathrm{F}_{\mathrm{G} 4}=10\left[\mathrm{AuL}_{3.5}\right]-26 \mathrm{~L}=\mathrm{Au}_{10} \mathrm{~L}_{9} ; \mathrm{S}=4 \mathrm{n}-12, \mathrm{Kp}=\mathrm{C}^{7} \mathrm{C}[\mathrm{M} 3]$

- $\mathrm{K}(\mathrm{n})=28(11): \mathrm{F}_{\mathrm{G} 5}=11\left[\mathrm{AuL}_{3.5}\right]-28 \mathrm{~L}=\mathrm{Au}_{11} \mathrm{~L}_{10.5} ; \mathrm{S}=4 \mathrm{n}-12, \mathrm{Kp}=\mathrm{C}^{7} \mathrm{C}[\mathrm{M} 4]$

- $\mathrm{K}(\mathrm{n})=30(12): \quad \mathrm{F}_{\mathrm{G} 6}=12\left[\mathrm{AuL}_{3.5}\right]-30 \mathrm{~L}=\mathrm{Au}_{12} \mathrm{~L}_{12} ; \mathrm{S}=4 \mathrm{n}-12, \mathrm{Kp}=\mathrm{C}^{7} \mathrm{C}[\mathrm{M} 5]$

- $\mathrm{K}(\mathrm{n})=32(13): \mathrm{F}_{\mathrm{G} 7}=13\left[\mathrm{AuL}_{3.5}\right]-32 \mathrm{~L}=\mathrm{Au}_{13} \mathrm{~L}_{11} ; \mathrm{S}=4 \mathrm{n}-12, \mathrm{Kp}=\mathrm{C}^{7} \mathrm{C}[\mathrm{M} 6]$

- $\mathrm{K}(\mathrm{n})=23(8): \quad \mathrm{F}_{\mathrm{G} 8}=8\left[\mathrm{AuL}_{3.5}\right]-23 \mathrm{~L}=\mathrm{Au}_{8} \mathrm{~L}_{5} ; \mathrm{S}=4 \mathrm{n}-14, \mathrm{Kp}=\mathrm{C}^{8} \mathrm{C}[\mathrm{M} 0]$

- $\mathrm{K}(\mathrm{n})=25(9): \quad \mathrm{F}_{\mathrm{G} 9}=9\left[\mathrm{AuL}_{3.5}\right]-25 \mathrm{~L}=\mathrm{Au}_{9} \mathrm{~L}_{6.5} ; \mathrm{S}=4 \mathrm{n}-14, \mathrm{Kp}=\mathrm{C}^{8} \mathrm{C}[\mathrm{M} 1]$

- $\mathrm{K}(\mathrm{n})=27(10): \mathrm{F}_{\mathrm{G} 10}=10\left[\mathrm{AuL}_{3.5}\right]-27 \mathrm{~L}=\mathrm{Au}_{10} \mathrm{~L}_{8} ; \mathrm{S}=4 \mathrm{n}-14, \mathrm{Kp}=\mathrm{C}^{8} \mathrm{C}[\mathrm{M} 2]$

- $\mathrm{K}(\mathrm{n})=29(11): \mathrm{F}_{\mathrm{G} 11}=11\left[\mathrm{AuL}_{3.5}\right]-29 \mathrm{~L}=\mathrm{Au}_{11} \mathrm{~L}_{9.5} ; \mathrm{S}=4 \mathrm{n}-14, \mathrm{Kp}=\mathrm{C}^{8} \mathrm{C}[\mathrm{M} 3]$

- $\mathrm{K}(\mathrm{n})=31(12): \mathrm{F}_{\mathrm{G} 12}=12\left[\mathrm{AuL}_{3.5}\right]-31 \mathrm{~L}=\mathrm{Au}_{12} \mathrm{~L}_{11} ; \mathrm{S}=4 \mathrm{n}-14, \mathrm{Kp}=\mathrm{C}^{8} \mathrm{C}[\mathrm{M} 4]$

- $\mathrm{K}(\mathrm{n})=33(13): \mathrm{F}_{\mathrm{G} 13}=13\left[\mathrm{AuL}_{3.5}\right]-33 \mathrm{~L}=\mathrm{Au}_{13} \mathrm{~L}_{12.5} ; \mathrm{S}=4 \mathrm{n}-14, \mathrm{Kp}=\mathrm{C}^{8} \mathrm{C}[\mathrm{M} 5]$

- $\mathrm{K}(\mathrm{n})=35(14): \mathrm{F}_{\mathrm{G} 14}=14\left[\mathrm{AuL}_{3.5}\right]-35 \mathrm{~L}=\mathrm{Au}_{14} \mathrm{~L}_{14} ; \mathrm{S}=4 \mathrm{n}-14, \mathrm{Kp}=\mathrm{C}^{8} \mathrm{C}[\mathrm{M} 6]$

HORIZONTAL MOVEMENT

When the type of series is kept constant and $\mathrm{K}$ value is increased by 2 , the capping remains the same but the nucleus expands. The series is kept constant by keeping $|2 \mathrm{n}-\mathrm{K}|=\mathrm{q} / 2$ constant. $\mid$

\section{VERTICAL MOVEMENT}

- $\quad[\mathrm{M} 1]$ series; $\mathrm{K}(\mathrm{n})=1(1): \mathrm{F}_{\mathrm{G} 15}=1\left[\mathrm{AuL}_{3.5}\right]-1 \mathrm{~L}=\mathrm{Au}_{1} \mathrm{~L}_{2.5} ; \mathrm{S}=4 \mathrm{n}+2, \mathrm{Kp}=\mathrm{C}^{0} \mathrm{C}[\mathrm{M} 1]$

- $\mathrm{K}(\mathrm{n})=1(1): \mathrm{F}_{\mathrm{G} 15}=1\left[\mathrm{AuL}_{3.5}\right]-1 \mathrm{~L}=\mathrm{Au}_{1} \mathrm{~L}_{2.5} ; \mathrm{S}=4 \mathrm{n}+2, \mathrm{Kp}=\mathrm{C}^{0} \mathrm{C}[\mathrm{M} 1]$

- $\mathrm{K}(\mathrm{n})=4(2): \mathrm{F}_{\mathrm{G} 16}=2\left[\mathrm{AuL}_{3.5}\right]-4 \mathrm{~L}=\mathrm{Au}_{2} \mathrm{~L}_{3} ; \mathrm{S}=4 \mathrm{n}+0, \mathrm{Kp}=\mathrm{C}^{1} \mathrm{C}[\mathrm{M} 1]$

- $\mathrm{K}(\mathrm{n})=7(3): \mathrm{F}_{\mathrm{G} 17}=3\left[\mathrm{AuL}_{3.5}\right]-7 \mathrm{~L}=\mathrm{Au}_{3} \mathrm{~L}_{3.5} ; \mathrm{S}=4 \mathrm{n}-2, \mathrm{Kp}=\mathrm{C}^{2} \mathrm{C}[\mathrm{M} 1]$

- $\mathrm{K}(\mathrm{n})=10(4): \mathrm{F}_{\mathrm{G} 18}=4\left[\mathrm{AuL}_{3.5}\right]-10 \mathrm{~L}=\mathrm{Au}_{4} \mathrm{~L}_{4} ; \mathrm{S}=4 \mathrm{n}-4, \mathrm{Kp}=\mathrm{C}^{3} \mathrm{C}[\mathrm{M} 1]$

- $\mathrm{K}(\mathrm{n})=13(5): \mathrm{F}_{\mathrm{G} 19}=5\left[\mathrm{AuL}_{3.5}\right]-13 \mathrm{~L}=\mathrm{Au}_{5} \mathrm{~L}_{4.5} ; \mathrm{S}=4 \mathrm{n}-6, \mathrm{Kp}=\mathrm{C}^{4} \mathrm{C}[\mathrm{M} 1]$

- $\mathrm{K}(\mathrm{n})=16(6): \mathrm{F}_{\mathrm{G} 20}=6\left[\mathrm{AuL}_{3.5}\right]-16 \mathrm{~L}=\mathrm{Au}_{6} \mathrm{~L}_{5} ; \mathrm{S}=4 \mathrm{n}-8, \mathrm{Kp}=\mathrm{C}^{5} \mathrm{C}[\mathrm{M} 1]$

- $\mathrm{K}(\mathrm{n})=19(7): \mathrm{F}_{\mathrm{G} 21}=7\left[\mathrm{AuL}_{3.5}\right]-19 \mathrm{~L}=\mathrm{Au}_{7} \mathrm{~L}_{5.5} ; \mathrm{S}=4 \mathrm{n}-10, \mathrm{Kp}=\mathrm{C}^{6} \mathrm{C}[\mathrm{M} 1]$

- $\mathrm{K}(\mathrm{n})=22(8): \mathrm{F}_{\mathrm{G} 22}=8\left[\mathrm{AuL}_{3.5}\right]-22 \mathrm{~L}=\mathrm{Au}_{8} \mathrm{~L}_{6} ; \mathrm{S}=4 \mathrm{n}-12, \mathrm{Kp}=\mathrm{C}^{7} \mathrm{C}[\mathrm{M} 1]$

- $\mathrm{K}(\mathrm{n})=25(9): \mathrm{F}_{\mathrm{G} 23}=9\left[\mathrm{AuL}_{3.5}\right]-25 \mathrm{~L}=\mathrm{Au}_{9} \mathrm{~L}_{6.5} ; \mathrm{S}=4 \mathrm{n}-14, \mathrm{Kp}=\mathrm{C}^{8} \mathrm{C}[\mathrm{M} 1]$

- $\mathrm{K}(\mathrm{n})=28(10): \mathrm{F}_{\mathrm{G} 24}=10\left[\mathrm{AuL}_{3.5}\right]-28 \mathrm{~L}=\mathrm{Au}_{10} \mathrm{~L}_{7} ; \mathrm{S}=4 \mathrm{n}-16, \mathrm{Kp}=\mathrm{C}^{9} \mathrm{C}[\mathrm{M} 1]$

- $\mathrm{K}(\mathrm{n})=31(11): \mathrm{F}_{\mathrm{G} 25}=11\left[\mathrm{AuL}_{3.5}\right]-31 \mathrm{~L}=\mathrm{Au}_{11} \mathrm{~L}_{7.5} ; \mathrm{S}=4 \mathrm{n}-18, \mathrm{Kp}=\mathrm{C}^{10} \mathrm{C}[\mathrm{M} 1]$

- $\mathrm{K}(\mathrm{n})=11(6): \mathrm{F}_{\mathrm{G} 26}=6\left[\mathrm{AuL}_{3.5}\right]-11 \mathrm{~L}=\mathrm{Au}_{6} \mathrm{~L}_{10} ; \mathrm{S}=4 \mathrm{n}+2, \mathrm{Kp}=\mathrm{C}^{0} \mathrm{C}[\mathrm{M} 6]$

- $\mathrm{K}(\mathrm{n})=14(7): \mathrm{F}_{\mathrm{G} 27}=7\left[\mathrm{AuL}_{3.5}\right]-14 \mathrm{~L}=\mathrm{Au}_{7} \mathrm{~L}_{10.5} ; \mathrm{S}=4 \mathrm{n}+0, \mathrm{Kp}=\mathrm{C}^{1} \mathrm{C}[\mathrm{M} 6]$

- $\mathrm{K}(\mathrm{n})=17(8): \mathrm{F}_{\mathrm{G} 28}=8\left[\mathrm{AuL}_{3.5}\right]-17 \mathrm{~L}=\mathrm{Au}_{8} \mathrm{~L}_{11} ; \mathrm{S}=4 \mathrm{n}-2, \mathrm{Kp}=\mathrm{C}^{2} \mathrm{C}[\mathrm{M} 6]$

- $\mathrm{K}(\mathrm{n})=20(9): \mathrm{F}_{\mathrm{G} 29}=9\left[\mathrm{AuL}_{3.5}\right]-20 \mathrm{~L}=\mathrm{Au}_{9} \mathrm{~L}_{11.5} ; \mathrm{S}=4 \mathrm{n}-4, \mathrm{Kp}=\mathrm{C}^{3} \mathrm{C}[\mathrm{M} 6]$

- $\mathrm{K}(\mathrm{n})=23(10): \mathrm{F}_{\mathrm{G} 30}=10\left[\mathrm{AuL}_{3.5}\right]-23 \mathrm{~L}=\mathrm{Au}_{10} \mathrm{~L}_{12} ; \mathrm{S}=4 \mathrm{n}-6, \mathrm{Kp}=\mathrm{C}^{4} \mathrm{C}[\mathrm{M} 6]$

- $\mathrm{K}(\mathrm{n})=26(11): \mathrm{F}_{\mathrm{G} 31}=11\left[\mathrm{AuL}_{3.5}\right]-26 \mathrm{~L}=\mathrm{Au}_{11} \mathrm{~L}_{12.5} ; \mathrm{S}=4 \mathrm{n}-8, \mathrm{Kp}=\mathrm{C}^{5} \mathrm{C}[\mathrm{M} 6]$

- $\mathrm{K}(\mathrm{n})=29(12): \mathrm{F}_{\mathrm{G} 32}=12\left[\mathrm{AuL}_{3.5}\right]-29 \mathrm{~L}=\mathrm{Au}_{12} \mathrm{~L}_{13} ; \mathrm{S}=4 \mathrm{n}-10, \mathrm{Kp}=\mathrm{C}^{6} \mathrm{C}[\mathrm{M} 6]$

- $\mathrm{K}(\mathrm{n})=32(13): \mathrm{F}_{\mathrm{G} 33}=13\left[\mathrm{AuL}_{3.5}\right]-32 \mathrm{~L}=\mathrm{Au}_{13} \mathrm{~L}_{13.5} ; \mathrm{S}=4 \mathrm{n}-12, \mathrm{Kp}=\mathrm{C}^{7} \mathrm{C}[\mathrm{M} 6]$

- $\mathrm{K}(\mathrm{n})=35(14): \mathrm{F}_{\mathrm{G} 34}=14\left[\mathrm{AuL}_{3.5}\right]-35 \mathrm{~L}=\mathrm{Au}_{14} \mathrm{~L}_{14} ; \mathrm{S}=4 \mathrm{n}-14, \mathrm{Kp}=\mathrm{C}^{8} \mathrm{C}[\mathrm{M} 6]$

- $\mathrm{K}(\mathrm{n})=38(15): \mathrm{F}_{\mathrm{G} 35}=15\left[\mathrm{AuL}_{3.5}\right]-38 \mathrm{~L}=\mathrm{Au}_{15} \mathrm{~L}_{14.5} ; \mathrm{S}=4 \mathrm{n}-16, \mathrm{Kp}=\mathrm{C}^{9} \mathrm{C}[\mathrm{M} 6]$

- $\mathrm{K}(\mathrm{n})=41(16): \mathrm{F}_{\mathrm{G} 36}=16\left[\mathrm{AuL}_{3.5}\right]-41 \mathrm{~L}=\mathrm{Au}_{16} \mathrm{~L}_{15} ; \mathrm{S}=4 \mathrm{n}-18, \mathrm{Kp}=\mathrm{C}^{10} \mathrm{C}[\mathrm{M} 6]$

As can be seen from the above examples, the vertical movement involves the increase in capping index while the nuclear size (nuclear index) remains the same. The calculation used in examples $F_{\mathrm{G} 1}$ to $\mathrm{F}_{\mathrm{G} 36}$ is the same applied in generating clusters in Table 2A and 2B. The clusters in Tables 2A and 2B cover the range $\mathrm{x}=-4$ to +4 , that is, [M-4] to [M4] and $\mathrm{n}$ values from 38-42. In Table 3, there is a collection of known clusters which have been categorized into their CLUSTER GROUPS. Furthermore, the K(n) values of the clusters were in agreement with those in Table 1 and their 
cluster formulas were also in agreement with the hypothetically derived ones in Tables $2 \mathrm{~A}$ and $2 \mathrm{~B}$. Finally Table 4 shows more of the reported golden clusters whose $K(n)$ values were calculated and then utilized together with the PRIMARY CLUSTER to generate the corresponding clusters for comparison with their parent clusters. It was found that both the parent clusters and their corresponding generated clusters we similar.

Table 3. The $K(n)$ Series for $M x, x=-6$ to +6

\begin{tabular}{|c|c|c|c|c|c|c|c|c|}
\hline $\begin{array}{l}\text { NUME } \\
\mathrm{T} 3\end{array}$ & RICAL & SERIES & & & & & Series & $\mathrm{Kp}$ \\
\hline 0 & 1 & 2 & 3 & 4 & 5 & 6 & & \\
\hline [M0] & [M1] & [M2] & [M3] & [M4] & [M5] & [M6] & & \\
\hline$-1(0)$ & 1(1) & $3(2)$ & $5(3)$ & $7(4)$ & $9(5)$ & $11(6)$ & $4 n+2$ & $\mathrm{C}^{0} \mathrm{C}[\mathrm{Mx}]$ \\
\hline 2(1) & $4(2)$ & $6(3)$ & $8(4)$ & $10(5)$ & $12(6)$ & $14(7)$ & $4 n+0$ & $\mathrm{C}^{1} \mathrm{C}[\mathrm{Mx}]$ \\
\hline $5(2)$ & $7(3)$ & $9(4)$ & $11(5)$ & $13(6)$ & $15(7)$ & $17(8)$ & $4 n-2$ & $\mathrm{C}^{2} \mathrm{C}[\mathrm{Mx}]$ \\
\hline $8(3)$ & $10(4)$ & $12(5)$ & $14(6)$ & $16(7)$ & $18(8)$ & 20(9) & $4 n-4$ & $\mathrm{C}^{3} \mathrm{C}[\mathrm{Mx}]$ \\
\hline $11(4)$ & 13(5) & $15(6)$ & $17(7)$ & $19(8)$ & $21(9)$ & $23(10)$ & $4 n-6$ & $\mathrm{C}^{4} \mathrm{C}[\mathrm{Mx}]$ \\
\hline $14(5)$ & $16(6)$ & $18(7)$ & $20(8)$ & $22(9)$ & $24(10)$ & $26(11)$ & $4 n-8$ & $\mathrm{C}^{5} \mathrm{C}[\mathrm{Mx}]$ \\
\hline $17(6)$ & 19(7) & $21(8)$ & $23(9)$ & $25(10)$ & $27(11)$ & $29(12)$ & $4 n-10$ & $\mathrm{C}^{6} \mathrm{C}[\mathrm{Mx}]$ \\
\hline $20(7)$ & $22(8)$ & $24(9)$ & $26(10)$ & $28(11)$ & $30(12)$ & $32(13)$ & $4 n-12$ & $\mathrm{C}^{7} \mathrm{C}[\mathrm{Mx}]$ \\
\hline $23(8)$ & $25(9)$ & $27(10)$ & 29(11) & 31(12) & 33(13) & $35(14)$ & $4 n-14$ & $\mathrm{C}^{8} \mathrm{C}[\mathrm{Mx}]$ \\
\hline $26(9)$ & $28(10)$ & $30(11)$ & $32(12)$ & $34(13)$ & $36(14)$ & $38(15)$ & $4 n-16$ & $\mathrm{C}^{9} \mathrm{C}[\mathrm{Mx}]$ \\
\hline $29(10)$ & 31(11) & $33(12)$ & $35(13)$ & $37(14)$ & $39(15)$ & 41(16) & $4 n-18$ & $\mathrm{C}^{10} \mathrm{C}[\mathrm{Mx}]$ \\
\hline $32(11)$ & 34(12) & $36(13)$ & $38(14)$ & $40(15)$ & $42(16)$ & $44(17)$ & $4 n-20$ & $\mathrm{C}^{11} \mathrm{C}[\mathrm{Mx}]$ \\
\hline $35(12)$ & 37(13) & $39(14)$ & $41(15)$ & $43(16)$ & $45(17)$ & $47(18)$ & $4 n-22$ & $\mathrm{C}^{12} \mathrm{C}[\mathrm{Mx}]$ \\
\hline $38(13)$ & $40(14)$ & $42(15)$ & $44(16)$ & $46(17)$ & $48(18)$ & $50(19)$ & $4 n-24$ & $\mathrm{C}^{13} \mathrm{C}[\mathrm{Mx}]$ \\
\hline $41(14)$ & $43(15)$ & $45(16)$ & $47(17)$ & $49(18)$ & $51(19)$ & $53(20)$ & $4 n-26$ & $\mathrm{C}^{14} \mathrm{C}[\mathrm{Mx}]$ \\
\hline $44(16)$ & $46(17)$ & $48(18)$ & $50(19)$ & $52(19)$ & $54(20)$ & $56(21)$ & $4 n-28$ & $\mathrm{C}^{15} \mathrm{C}[\mathrm{Mx}]$ \\
\hline $47(16)$ & $49(17)$ & $51(18)$ & $53(19)$ & $55(20)$ & $57(21)$ & $59(22)$ & $4 n-30$ & $\mathrm{C}^{16} \mathrm{C}[\mathrm{Mx}]$ \\
\hline $50(17)$ & $52(18)$ & $54(19)$ & $56(20)$ & $58(21)$ & $60(22)$ & $62(23)$ & $4 n-32$ & $\mathrm{C}^{17} \mathrm{C}[\mathrm{Mx}]$ \\
\hline $53(18)$ & $55(19)$ & $57(20)$ & $59(21)$ & $61(22)$ & $63(23)$ & $65(24)$ & $4 n-34$ & $\mathrm{C}^{18} \mathrm{C}[\mathrm{Mx}]$ \\
\hline $56(19)$ & $58(20)$ & $60(21)$ & $62(22)$ & $64(23)$ & $66(24)$ & $68(25)$ & $4 n-36$ & $\mathrm{C}^{19} \mathrm{C}[\mathrm{Mx}]$ \\
\hline $59(20)$ & $61(21)$ & $63(22)$ & $65(23)$ & $67(24)$ & $69(25)$ & $71(26)$ & $4 n-38$ & $\mathrm{C}^{20} \mathrm{C}[\mathrm{Mx}]$ \\
\hline $62(21)$ & $64(22)$ & $66(23)$ & $68(24)$ & $70(25)$ & $72(26)$ & $74(27)$ & $4 n-40$ & $\mathrm{C}^{21} \mathrm{C}[\mathrm{Mx}]$ \\
\hline $65(22)$ & $67(23)$ & $69(24)$ & $71(25)$ & $73(26)$ & $75(27)$ & $77(28)$ & $4 n-42$ & $\mathrm{C}^{22} \mathrm{C}[\mathrm{Mx}]$ \\
\hline $68(23)$ & $70(24)$ & $72(25)$ & $74(26)$ & $76(27)$ & $78(28)$ & $80(29)$ & $4 n-44$ & $\mathrm{C}^{23} \mathrm{C}[\mathrm{Mx}]$ \\
\hline
\end{tabular}

Table 3. Continued

\begin{tabular}{|c|c|c|c|c|c|c|c|}
\hline $\begin{array}{l}\text { NUME } \\
\text { T3 }\end{array}$ & RICAL & SERIES & & & & & \\
\hline-6 & -5 & -4 & -3 & -2 & -1 & & \\
\hline [M-6] & [M-5] & [M-4] & [M-3] & [M-2] & [M-1] & Series & $\mathrm{Kp}$ \\
\hline$-13(-6)$ & $-11(-5)$ & $-9(-4)$ & $-7(-3)$ & $-5(-2)$ & $-3(-1)$ & $4 n+2$ & $\mathrm{C}^{0} \mathrm{C}[\mathrm{Mx}]$ \\
\hline$-10(-5)$ & $-8(-4)$ & $-6(-3)$ & $-4(-2)$ & $-2(-1)$ & $0(0)$ & $4 n+0$ & $\mathrm{C}^{1} \mathrm{C}[\mathrm{Mx}]$ \\
\hline$-7(-4)$ & $-5(-3)$ & $-3(-2)$ & $-1(-1)$ & $1(0)$ & $3(1)$ & $4 n-2$ & $\mathrm{C}^{2} \mathrm{C}[\mathrm{Mx}]$ \\
\hline$-4(-3)$ & $-2(-2)$ & $0(-1)$ & $2(0)$ & $4(1)$ & $6(2)$ & $4 n-4$ & $\mathrm{C}^{3} \mathrm{C}[\mathrm{Mx}]$ \\
\hline$-1(-2)$ & $1(-1)$ & $3(0)$ & $5(1)$ & $7(2)$ & $9(3)$ & $4 n-6$ & $\mathrm{C}^{4} \mathrm{C}[\mathrm{Mx}]$ \\
\hline $2(-1)$ & $4(0)$ & $6(1)$ & $8(2)$ & $10(3)$ & $12(4)$ & $4 n-8$ & $\mathrm{C}^{5} \mathrm{C}[\mathrm{Mx}]$ \\
\hline $5(0)$ & $7(1)$ & $9(2)$ & 11(3) & $13(4)$ & $15(5)$ & $4 n-10$ & $\mathrm{C}^{6} \mathrm{C}[\mathrm{Mx}]$ \\
\hline $8(3)$ & $10(2)$ & $12(3)$ & 14(4) & $16(5)$ & $18(6)$ & $4 n-12$ & $\mathrm{C}^{7} \mathrm{C}[\mathrm{Mx}]$ \\
\hline $11(2)$ & 13(3) & $15(4)$ & $17(5)$ & 19(6) & $21(7)$ & $4 n-14$ & $\mathrm{C}^{8} \mathrm{C}[\mathrm{Mx}]$ \\
\hline $14(3)$ & $16(4)$ & $18(5)$ & $20(6)$ & $22(7)$ & $24(8)$ & $4 n-16$ & $\mathrm{C}^{9} \mathrm{C}[\mathrm{Mx}]$ \\
\hline $17(4)$ & $19(5)$ & $21(6)$ & $23(7)$ & $25(8)$ & $27(9)$ & $4 n-18$ & $\mathrm{C}^{10} \mathrm{C}[\mathrm{Mx}]$ \\
\hline $20(5)$ & $22(6)$ & $24(7)$ & $26(8)$ & $28(9)$ & $30(10)$ & $4 n-20$ & $\mathrm{C}^{11} \mathrm{C}[\mathrm{Mx}]$ \\
\hline $23(6)$ & $25(7)$ & $27(8)$ & $29(9)$ & $31(10)$ & $33(11)$ & $4 n-22$ & $\mathrm{C}^{12} \mathrm{C}[\mathrm{Mx}]$ \\
\hline $26(7)$ & $28(8)$ & $30(9$ & $32(10)$ & $34(11)$ & $36(12)$ & $4 n-24$ & $\mathrm{C}^{13} \mathrm{C}[\mathrm{Mx}]$ \\
\hline $29(8)$ & $31(9)$ & $33(10)$ & $35(11)$ & $37(12)$ & $39(13)$ & $4 n-26$ & $\mathrm{C}^{14} \mathrm{C}[\mathrm{Mx}]$ \\
\hline $32(9)$ & $34(10)$ & $36(11)$ & $38(12)$ & $40(13)$ & $42(14)$ & $4 n-28$ & $\mathrm{C}^{15} \mathrm{C}[\mathrm{Mx}]$ \\
\hline $35(10)$ & $37(11)$ & $39(12)$ & $41(13)$ & $43(14)$ & $45(15)$ & $4 n-30$ & $\mathrm{C}^{16} \mathrm{C}[\mathrm{Mx}]$ \\
\hline $38(11)$ & $40(12)$ & $42(13)$ & $44(14)$ & $46(15)$ & $48(16)$ & $4 n-32$ & $\mathrm{C}^{17} \mathrm{C}[\mathrm{Mx}]$ \\
\hline $41(12)$ & $43(13)$ & $45(14)$ & $47(15)$ & $49(16)$ & $51(17)$ & $4 n-34$ & $\mathrm{C}^{18} \mathrm{C}[\mathrm{Mx}]$ \\
\hline $44(13)$ & $46(14)$ & $48(15)$ & $50(16)$ & $52(17)$ & $54(18)$ & $4 n-36$ & $\mathrm{C}^{19} \mathrm{C}[\mathrm{Mx}]$ \\
\hline $47(14)$ & $49(15)$ & $51(16)$ & $53(17)$ & $55(18)$ & $57(19)$ & $4 n-38$ & $\mathrm{C}^{20} \mathrm{C}[\mathrm{Mx}]$ \\
\hline $50(15)$ & $52(16)$ & $54(17)$ & $56(18)$ & $58(19)$ & $60(20)$ & $4 n-40$ & $\mathrm{C}^{21} \mathrm{C}[\mathrm{Mx}]$ \\
\hline $53(16)$ & $55(17)$ & $57(18)$ & $59(19)$ & $61(20)$ & $63(21)$ & $4 n-42$ & $\mathrm{C}^{22} \mathrm{C}[\mathrm{Mx}]$ \\
\hline $56(17)$ & $58(18)$ & $60(19)$ & $62(20)$ & $64(21)$ & $66(22)$ & $4 n-44$ & $\mathrm{C}^{23} \mathrm{C}[\mathrm{Mx}]$ \\
\hline $59(18)$ & 61(19) & $63(20)$ & $65(21)$ & $67(22)$ & $69(23)$ & $4 n-46$ & $\mathrm{C}^{24} \mathrm{C}[\mathrm{Mx}]$ \\
\hline $62(19)$ & $64(20)$ & $66(21)$ & $68(22)$ & $70(23)$ & $72(24)$ & $4 n-48$ & $\mathrm{C}^{25} \mathrm{C}[\mathrm{Mx}]$ \\
\hline
\end{tabular}


Table 3. Continued

\begin{tabular}{|c|c|c|c|c|c|c|c|}
\hline NUME & RICAL & SERIES & & & & & \\
\hline-6 & -5 & -4 & -3 & -2 & -1 & & \\
\hline $65(20)$ & $67(21)$ & $69(22)$ & $71(23)$ & $73(24)$ & $75(25)$ & $4 n-50$ & $\mathrm{C}^{25} \mathrm{C}[\mathrm{Mx}]$ \\
\hline $68(21)$ & $70(22)$ & $72(23)$ & $74(24)$ & $76(25)$ & $78(26)$ & $4 n-52$ & $\mathrm{C}^{27} \mathrm{C}[\mathrm{Mx}]$ \\
\hline $71(22)$ & $73(23)$ & $75(24)$ & $77(25)$ & $79(26)$ & $81(27)$ & $4 n-54$ & $\mathrm{C}^{28} \mathrm{C}[\mathrm{Mx}]$ \\
\hline $74(23)$ & $76(24)$ & $78(25)$ & $80(26)$ & $82(27)$ & $84(28)$ & $4 n-56$ & $\mathrm{C}^{29} \mathrm{C}[\mathrm{Mx}]$ \\
\hline $77(24)$ & $79(25)$ & $81(26)$ & $83(27)$ & $85(28)$ & $87(29)$ & $4 n-58$ & $\mathrm{C}^{30} \mathrm{C}[\mathrm{Mx}]$ \\
\hline $80(25)$ & $82(26)$ & $84(27)$ & $86(28)$ & $88(29)$ & $90(30)$ & $4 n-60$ & $\mathrm{C}^{31} \mathrm{C}[\mathrm{Mx}]$ \\
\hline $83(26)$ & $85(27)$ & $87(28)$ & $89(29)$ & $91(30)$ & $93(31)$ & $4 n-62$ & $\mathrm{C}^{32} \mathrm{C}[\mathrm{Mx}]$ \\
\hline $86(27)$ & $88(28)$ & $90(29)$ & $92(30)$ & $94(31)$ & $96(32)$ & $4 n-64$ & $\mathrm{C}^{33} \mathrm{C}[\mathrm{Mx}]$ \\
\hline $89(28)$ & $91(29)$ & $93(30)$ & $95(31)$ & $97(32)$ & $99(33)$ & $4 n-66$ & $\mathrm{C}^{34} \mathrm{C}[\mathrm{Mx}]$ \\
\hline $92(29)$ & $94(30)$ & $96(31)$ & $98(32)$ & $100(33)$ & $102(34)$ & $4 n-68$ & $\mathrm{C}^{35} \mathrm{C}[\mathrm{Mx}]$ \\
\hline $95(30)$ & $97(31)$ & $99(32)$ & 101(33) & 103(34) & $105(35)$ & $4 n-70$ & $\mathrm{C}^{36} \mathrm{C}[\mathrm{Mx}]$ \\
\hline $98(31)$ & $100(32)$ & $102(33)$ & 104(34) & $106(35)$ & $108(36)$ & $4 n-72$ & $\mathrm{C}^{37} \mathrm{C}[\mathrm{Mx}]$ \\
\hline 101(32) & $103(33)$ & $105(34)$ & $107(35)$ & $109(36)$ & 111(37) & $4 n-74$ & $\mathrm{C}^{38} \mathrm{C}[\mathrm{Mx}]$ \\
\hline 104(33) & $106(34)$ & $108(35)$ & $110(36)$ & $112(37)$ & $114(38)$ & $4 n-76$ & $\mathrm{C}^{39} \mathrm{C}[\mathrm{Mx}]$ \\
\hline $107(34)$ & $109(35)$ & $111(36)$ & 113(37) & $115(38)$ & $117(39)$ & $4 n-78$ & $\mathrm{C}^{40} \mathrm{C}[\mathrm{Mx}]$ \\
\hline $110(35)$ & $112(36)$ & $114(37)$ & $116(38)$ & $118(39)$ & $120(40)$ & $4 n-80$ & $\mathrm{C}^{41} \mathrm{C}[\mathrm{Mx}]$ \\
\hline $113(36)$ & $115(37)$ & $117(38)$ & 119(39) & $121(40)$ & $123(41)$ & $4 n-82$ & $\mathrm{C}^{42} \mathrm{C}[\mathrm{Mx}]$ \\
\hline $116(37)$ & $118(38)$ & $120(39)$ & $122(40)$ & $124(41)$ & $126(42)$ & $4 n-84$ & $\mathrm{C}^{43} \mathrm{C}[\mathrm{Mx}]$ \\
\hline
\end{tabular}

Table 4. Derived Golden Clusters Using K(n) Parameter

\begin{tabular}{|c|c|c|c|c|c|}
\hline $\begin{array}{l}\text { HYPOT } \\
\text { T4 }\end{array}$ & HETICAL & DERIVA & TIONS & & \\
\hline [M0] & {$\left[\mathrm{AuL}_{3.5}\right]$} & [M1] & {$\left[\mathrm{AuL}_{3.5}\right]$} & [M2] & {$\left[\mathrm{AuL}_{3.5}\right]$} \\
\hline K(n) & [M0] & K(n) & [M1] & $K(n)$ & [M2] \\
\hline$-1(0)$ & $-1 \mathrm{~L}$ & 1(1) & $\mathrm{AuL}_{2.5}$ & $3(2)$ & $\mathrm{Au}_{2} \mathrm{~L}_{4}$ \\
\hline $2(1)$ & $\mathrm{AuL}_{15}$ & $4(2)$ & $\mathrm{Au}_{2} \mathrm{~L}_{3}$ & $6(3)$ & $\mathrm{Au}_{3} \mathrm{~L}_{45}$ \\
\hline $5(2)$ & $\mathrm{Au}_{2} \mathrm{~L}_{2}$ & $7(3)$ & $\mathrm{Au}_{3} \mathrm{~L}_{3.5}$ & $9(4)$ & $\mathrm{Au}_{4} \mathrm{~L}_{5}$ \\
\hline $8(3)$ & $\mathrm{Au}_{3} \mathrm{~L}_{2.5}$ & $10(4)$ & $\mathrm{Au}_{4} \mathrm{~L}_{4}$ & $12(5)$ & $\mathrm{Au}_{5} \mathrm{~L}_{5.5}$ \\
\hline $11(4)$ & $\mathrm{Au}_{4} \mathrm{~L}_{3}$ & $13(5)$ & $\mathrm{Au}_{5} \mathrm{~L}_{4.5}$ & $15(6)$ & $\mathrm{Au}_{6} \mathrm{~L}_{6}$ \\
\hline $14(5)$ & $\mathrm{Au}_{5} \mathrm{~L}_{3.5}$ & $16(6)$ & $\mathrm{Au}_{6} \mathrm{~L}_{5}$ & $18(7)$ & $\mathrm{Au}_{7} \mathrm{~L}_{6.5}$ \\
\hline $17(6)$ & $\mathrm{Au}_{6} \mathrm{~L}_{4}$ & 19(7) & $\mathrm{Au}_{7} \mathrm{~L}_{5.5}$ & $21(8)$ & $\mathrm{Au}_{8} \mathrm{~L}_{7}$ \\
\hline $20(7)$ & $\mathrm{Au}_{7} \mathrm{~L}_{4.5}$ & $22(8)$ & $\mathrm{Au}_{8} \mathrm{~L}_{6}$ & $24(9)$ & $\mathrm{Au}_{9} \mathrm{~L}_{7.5}$ \\
\hline $23(8)$ & $\mathrm{Au}_{8} \mathrm{~L}_{5}$ & $25(9)$ & $\mathrm{Au}_{9} \mathrm{~L}_{6.5}$ & $27(10)$ & $\mathrm{Au}_{10} \mathrm{~L}_{8}$ \\
\hline $26(9)$ & $\mathrm{Au}_{9} \mathrm{~L}_{5.5}$ & $28(10)$ & $\mathrm{Au}_{10} \mathrm{~L}_{7}$ & $30(11)$ & $\mathrm{Au}_{11} \mathrm{~L}_{8.5}$ \\
\hline $29(10)$ & $\mathrm{Au}_{10} \mathrm{~L}_{6}$ & $31(11)$ & $\mathrm{Au}_{11} \mathrm{~L}_{7.5}$ & $33(12)$ & $\mathrm{Au}_{12} \mathrm{~L}_{9}$ \\
\hline $32(11)$ & $\mathrm{Au}_{11} \mathrm{~L}_{65}$ & $34(12)$ & $\mathrm{Au}_{12} \mathrm{~L}_{8}$ & $36(13)$ & $\mathrm{Au}_{13} \mathrm{~L}_{95}$ \\
\hline $35(12)$ & $\mathrm{Au}_{12} \mathrm{~L}_{7}$ & $37(13)$ & $\mathrm{Au}_{13} \mathrm{~L}_{8.5}$ & $39(14)$ & $\mathrm{Au}_{14} \mathrm{~L}_{10}$ \\
\hline $38(13)$ & $\mathrm{Au}_{13} \mathrm{~L}_{7.5}$ & $40(14)$ & $\mathrm{Au}_{14} \mathrm{~L}_{9}$ & $42(15)$ & $\mathrm{Au}_{15} \mathrm{~L}_{10.5}$ \\
\hline $41(14)$ & $\mathrm{Au}_{14} \mathrm{~L}_{8}$ & $43(15)$ & $\mathrm{Au}_{15} \mathrm{~L}_{9.5}$ & $45(16)$ & $\mathrm{Au}_{16} \mathrm{~L}_{11}$ \\
\hline $44(15)$ & $\mathrm{Au}_{15} \mathrm{~L}_{85}$ & $46(16)$ & $\mathrm{Au}_{16} \mathrm{~L}_{10}$ & $48(17)$ & $\mathrm{Au}_{17} \mathrm{~L}_{115}$ \\
\hline $47(16)$ & $\mathrm{Au}_{16} \mathrm{~L}_{9}$ & $49(17)$ & $\mathrm{Au}_{17} \mathrm{~L}_{10.5}$ & $51(18)$ & $\mathrm{Au}_{18} \mathrm{~L}_{12}$ \\
\hline $50(17)$ & $\mathrm{Au}_{17} \mathrm{~L}_{9.5}$ & $52(18)$ & $\mathrm{Au}_{18} \mathrm{~L}_{11}$ & $54(19)$ & $\mathrm{Au}_{19} \mathrm{~L}_{12.5}$ \\
\hline $53(18)$ & $\mathrm{Au}_{18} \mathrm{~L}_{10}$ & $55(19)$ & $\mathrm{Au}_{19} \mathrm{~L}_{11.5}$ & $57(20)$ & $\mathrm{Au}_{20} \mathrm{~L}_{13}$ \\
\hline $56(19)$ & $\mathrm{Au}_{19} \mathrm{~L}_{10.5}$ & $58(20)$ & $\mathrm{Au}_{20} \mathrm{~L}_{12}$ & $60(21)$ & $\mathrm{Au}_{21} \mathrm{~L}_{13.5}$ \\
\hline $59(20)$ & $\mathrm{Au}_{20} \mathrm{~L}_{11}$ & $61(21)$ & $\mathrm{Au}_{21} \mathrm{~L}_{12.5}$ & $63(22)$ & $\mathrm{Au}_{22} \mathrm{~L}_{14}$ \\
\hline $62(21)$ & $\mathrm{Au}_{21} \mathrm{~L}_{11.5}$ & $64(22)$ & $\mathrm{Au}_{22} \mathrm{~L}_{13}$ & $66(23)$ & $\mathrm{Au}_{23} \mathrm{~L}_{14.5}$ \\
\hline $65(22)$ & $\mathrm{Au}_{22} \mathrm{~L}_{12}$ & $67(23)$ & $\mathrm{Au}_{23} \mathrm{~L}_{13.5}$ & $69(24)$ & $\mathrm{Au}_{24} \mathrm{~L}_{15}$ \\
\hline $68(23)$ & $\mathrm{Au}_{23} \mathrm{~L}_{12.5}$ & $70(24)$ & $\mathrm{Au}_{24} \mathrm{~L}_{14}$ & $72(25)$ & $\mathrm{Au}_{25} \mathrm{~L}_{15.5}$ \\
\hline $71(24)$ & $\mathrm{Au}_{24} \mathrm{~L}_{13}$ & $73(25)$ & $\mathrm{Au}_{25} \mathrm{~L}_{14.5}$ & $75(26)$ & $\mathrm{Au}_{26} \mathrm{~L}_{16}$ \\
\hline $74(25)$ & $\mathrm{Au}_{25} \mathrm{~L}_{13.5}$ & $76(26)$ & $\mathrm{Au}_{26} \mathrm{~L}_{15}$ & $78(27)$ & $\mathrm{Au}_{27} \mathrm{~L}_{16.5}$ \\
\hline $77(26)$ & $\mathrm{Au}_{26} \mathrm{~L}_{14}$ & $79(27)$ & $\mathrm{Au}_{27} \mathrm{~L}_{15.5}$ & $81(28)$ & $\mathrm{Au}_{28} \mathrm{~L}_{17}$ \\
\hline $80(27)$ & $\mathrm{Au}_{27} \mathrm{~L}_{14.5}$ & $82(28)$ & $\mathrm{Au}_{28} \mathrm{~L}_{16}$ & $84(29)$ & $\mathrm{Au}_{29} \mathrm{~L}_{17.5}$ \\
\hline $83(28)$ & $\mathrm{Au}_{28} \mathrm{~L}_{15}$ & $85(29)$ & $\mathrm{Au}_{29} \mathrm{~L}_{16.5}$ & $87(30)$ & $\mathrm{Au}_{30} \mathrm{~L}_{18}$ \\
\hline $86(29)$ & $\mathrm{Au}_{29} \mathrm{~L}_{15.5}$ & $88(30)$ & $\mathrm{Au}_{30} \mathrm{~L}_{17}$ & $90(31)$ & $\mathrm{Au}_{31} \mathrm{~L}_{18.5}$ \\
\hline $89(30)$ & $\mathrm{Au}_{30} \mathrm{~L}_{16}$ & $91(31)$ & $\mathrm{Au}_{31} \mathrm{~L}_{17.5}$ & $92(32)$ & $\mathrm{Au}_{32} \mathrm{~L}_{19}$ \\
\hline $92(31)$ & $\mathrm{Au}_{31} \mathrm{~L}_{16.5}$ & $84(32)$ & $\mathrm{Au}_{32} \mathrm{~L}_{18}$ & $96(33)$ & $\mathrm{Au}_{33} \mathrm{~L}_{19.5}$ \\
\hline $95(32)$ & $\mathrm{Au}_{32} \mathrm{~L}_{17}$ & $97(33)$ & $\mathrm{Au}_{33} \mathrm{~L}_{18.5}$ & $99(34)$ & $\mathrm{Au}_{34} \mathrm{~L}_{20}$ \\
\hline $98(33)$ & $\mathrm{Au}_{33} \mathrm{~L}_{17.5}$ & $100(34)$ & $\mathrm{Au}_{34} \mathrm{~L}_{19}$ & $102(35)$ & $\mathrm{Au}_{35} \mathrm{~L}_{20.5}$ \\
\hline 101(34) & $\mathrm{Au}_{34} \mathrm{~L}_{18}$ & $103(35)$ & $\mathrm{Au}_{35} \mathrm{~L}_{19,5}$ & $105(36)$ & $\mathrm{Au}_{36} \mathrm{~L}_{21}$ \\
\hline $104(35)$ & $\mathrm{Au}_{35} \mathrm{~L}_{18.5}$ & $106(36)$ & $\mathrm{Au}_{36} \mathrm{~L}_{20}$ & $108(37)$ & $\mathrm{Au}_{37} \mathrm{~L}_{21.5}$ \\
\hline $107(36)$ & $\mathrm{Au}_{36} \mathrm{~L}_{19}$ & $109(37)$ & $\mathrm{Au}_{37} \mathrm{~L}_{20.5}$ & 111(38) & $\mathrm{Au}_{38} \mathrm{~L}_{22}$ \\
\hline $\mathrm{K}(\mathrm{n})$ & [M0] & $\mathrm{K}(\mathrm{n})$ & [M1] & $\mathrm{K}(\mathrm{n})$ & [M2] \\
\hline $110(37)$ & $\mathrm{Au}_{37} \mathrm{~L}_{19.5}$ & $112(38)$ & $\mathrm{Au}_{38} \mathrm{~L}_{21}$ & $114(39)$ & $\mathrm{Au}_{39} \mathrm{~L}_{22.5}$ \\
\hline $113(38)$ & $\mathrm{Au}_{38} \mathrm{~L}_{20}$ & $115(39)$ & $\mathrm{Au}_{39} \mathrm{~L}_{21.5}$ & $117(40)$ & $\mathrm{Au}_{40} \mathrm{~L}_{23}$ \\
\hline 116(39) & $\mathrm{Au}_{39} \mathrm{~L}_{205}$ & $118(40)$ & $\mathrm{Au}_{40} \mathrm{~L}_{22}$ & $120(41)$ & $\mathrm{Au}_{41} \mathrm{~L}_{23.5}$ \\
\hline $119(40)$ & $\mathrm{Au}_{40} \mathrm{~L}_{21}$ & $121(41)$ & $\mathrm{Au}_{41} \mathrm{~L}_{22.5}$ & $123(42)$ & $\mathrm{Au}_{42} \mathrm{~L}_{24}$ \\
\hline $122(41)$ & $\mathrm{Au}_{41} \mathrm{~L}_{21.5}$ & $124(42)$ & $\mathrm{Au}_{42} \mathrm{~L}_{23}$ & $126(43)$ & $\mathrm{Au}_{43} \mathrm{~L}_{24.5}$ \\
\hline $125(42)$ & $\mathrm{Au}_{42} \mathrm{~L}_{22}$ & $127(43)$ & $\mathrm{Au}_{43} \mathrm{~L}_{23.5}$ & $129(44)$ & $\mathrm{Au}_{44} \mathrm{~L}_{25}$ \\
\hline
\end{tabular}


Table 4. Continued

\begin{tabular}{|c|c|c|c|}
\hline $\begin{array}{l}\mathrm{T} 4 \\
{[\mathrm{M} 3]}\end{array}$ & {$\left[\mathrm{AuL}_{3.5}\right]$} & [M4] & {$\left[\mathrm{AuL}_{3.5}\right]$} \\
\hline$K(n)$ & [M3] & K(n) & [M4] \\
\hline $5(3)$ & $\mathrm{Au}_{3} \mathrm{~L}_{5.5}$ & $7(4)$ & $\mathrm{Au}_{4} \mathrm{~L}_{7}$ \\
\hline $8(4)$ & $\mathrm{Au}_{4} \mathrm{~L}_{6}$ & $10(5)$ & $\mathrm{Au}_{5} \mathrm{~L}_{7.5}$ \\
\hline 11(5) & $\mathrm{Au}_{5} \mathrm{~L}_{6.5}$ & $13(6)$ & $\mathrm{Au}_{6} \mathrm{~L}_{8}$ \\
\hline $14(6)$ & $\mathrm{Au}_{6} \mathrm{~L}_{7}$ & $16(7)$ & $\mathrm{Au}_{7} \mathrm{~L}_{8.5}$ \\
\hline 17(7) & $\mathrm{Au}_{7} \mathrm{~L}_{7.5}$ & $19(8)$ & $\mathrm{Au}_{8} \mathrm{~L}_{9}$ \\
\hline $20(8)$ & $\mathrm{Au}_{8} \mathrm{~L}_{8}$ & $22(9)$ & $\mathrm{Au}_{9} \mathrm{~L}_{9.5}$ \\
\hline $23(9)$ & $\mathrm{Au}_{9} \mathrm{~L}_{8.5}$ & $25(10)$ & $\mathrm{Au}_{10} \mathrm{~L}_{10}$ \\
\hline $26(10)$ & $\mathrm{Au}_{10} \mathrm{~L}_{9}$ & $28(11)$ & $\mathrm{Au}_{11} \mathrm{~L}_{10.5}$ \\
\hline $29(11)$ & $\mathrm{Au}_{11} \mathrm{~L}_{9.5}$ & $31(12)$ & $\mathrm{Au}_{12} \mathrm{~L}_{12}$ \\
\hline $32(12)$ & $\mathrm{Au}_{12} \mathrm{~L}_{10}$ & $34(13)$ & $\mathrm{Au}_{13} \mathrm{~L}_{12.5}$ \\
\hline $35(13)$ & $\mathrm{Au}_{13} \mathrm{~L}_{10.5}$ & $37(14)$ & $\mathrm{Au}_{14} \mathrm{~L}_{13}$ \\
\hline $38(14)$ & $\mathrm{Au}_{14} \mathrm{~L}_{11}$ & $40(15)$ & $\mathrm{Au}_{15} \mathrm{~L}_{13.5}$ \\
\hline 41(15) & $\mathrm{Au}_{15} \mathrm{~L}_{11.5}$ & $43(16)$ & $\mathrm{Au}_{16} \mathrm{~L}_{14}$ \\
\hline $44(16)$ & $\mathrm{Au}_{16} \mathrm{~L}_{12}$ & $46(17)$ & $\mathrm{Au}_{17} \mathrm{~L}_{14.5}$ \\
\hline $47(17)$ & $\mathrm{Au}_{17} \mathrm{~L}_{12.5}$ & $49(18)$ & $\mathrm{Au}_{18} \mathrm{~L}_{15}$ \\
\hline $50(18)$ & $\mathrm{Au}_{18} \mathrm{~L}_{13}$ & $52(19)$ & $\mathrm{Au}_{19} \mathrm{~L}_{15.5}$ \\
\hline $53(19)$ & $\mathrm{Au}_{19} \mathrm{~L}_{13.5}$ & $55(20)$ & $\mathrm{Au}_{20} \mathrm{~L}_{16}$ \\
\hline $56(20)$ & $\mathrm{Au}_{20} \mathrm{~L}_{14}$ & $58(21)$ & $\mathrm{Au}_{21} \mathrm{~L}_{16.5}$ \\
\hline $59(21)$ & $\mathrm{Au}_{21} \mathrm{~L}_{14.5}$ & $61(22)$ & $\mathrm{Au}_{22} \mathrm{~L}_{17}$ \\
\hline $62(22)$ & $\mathrm{Au}_{22} \mathrm{~L}_{15}$ & $64(23)$ & $\mathrm{Au}_{23} \mathrm{~L}_{17.5}$ \\
\hline $65(23)$ & $\mathrm{Au}_{23} \mathrm{~L}_{15.5}$ & $67(24)$ & $\mathrm{Au}_{24} \mathrm{~L}_{18}$ \\
\hline $68(24)$ & $\mathrm{Au}_{24} \mathrm{~L}_{16}$ & $70(25)$ & $\mathrm{Au}_{25} \mathrm{~L}_{18.5}$ \\
\hline $71(25)$ & $\mathrm{Au}_{25} \mathrm{~L}_{16.5}$ & $73(26)$ & $\mathrm{Au}_{26} \mathrm{~L}_{19}$ \\
\hline $74(26)$ & $\mathrm{Au}_{26} \mathrm{~L}_{17}$ & $76(27)$ & $\mathrm{Au}_{27} \mathrm{~L}_{19.5}$ \\
\hline $77(27)$ & $\mathrm{Au}_{27} \mathrm{~L}_{17.5}$ & $79(28)$ & $\mathrm{Au}_{28} \mathrm{~L}_{20}$ \\
\hline $80(28)$ & $\mathrm{Au}_{28} \mathrm{~L}_{18}$ & $82(29)$ & $\mathrm{Au}_{29} \mathrm{~L}_{20.5}$ \\
\hline $83(29)$ & $\mathrm{Au}_{29} \mathrm{~L}_{18.5}$ & $85(30)$ & $\mathrm{Au}_{30} \mathrm{~L}_{21}$ \\
\hline $86(30)$ & $\mathrm{Au}_{30} \mathrm{~L}_{19}$ & $88(31)$ & $\mathrm{Au}_{31} \mathrm{~L}_{21.5}$ \\
\hline $89(31)$ & $\mathrm{Au}_{31} \mathrm{~L}_{19.5}$ & $91(32)$ & $\mathrm{Au}_{32} \mathrm{~L}_{22}$ \\
\hline $92(32)$ & $\mathrm{Au}_{32} \mathrm{~L}_{20}$ & $94(33)$ & $\mathrm{Au}_{33} \mathrm{~L}_{22.5}$ \\
\hline $95(33)$ & $\mathrm{Au}_{33} \mathrm{~L}_{20.5}$ & $97(34)$ & $\mathrm{Au}_{34} \mathrm{~L}_{23}$ \\
\hline $98(34)$ & $\mathrm{Au}_{34} \mathrm{~L}_{21}$ & $100(35)$ & $\mathrm{Au}_{35} \mathrm{~L}_{23.5}$ \\
\hline 101(35) & $\mathrm{Au}_{35} \mathrm{~L}_{21.5}$ & $103(36)$ & $\mathrm{Au}_{36} \mathrm{~L}_{24}$ \\
\hline 104(36) & $\mathrm{Au}_{36} \mathrm{~L}_{22}$ & 106(37) & $\mathrm{Au}_{37} \mathrm{~L}_{24.5}$ \\
\hline 107(37) & $\mathrm{Au}_{37} \mathrm{~L}_{22.5}$ & $109(38)$ & $\mathrm{Au}_{38} \mathrm{~L}_{25}$ \\
\hline $110(38)$ & $\mathrm{Au}_{38} \mathrm{~L}_{23}$ & $112(39)$ & $\mathrm{Au}_{39} \mathrm{~L}_{25.5}$ \\
\hline 113(39) & $\mathrm{Au}_{39} \mathrm{~L}_{23.5}$ & $115(40)$ & $\mathrm{Au}_{40} \mathrm{~L}_{26}$ \\
\hline $116(40)$ & $\mathrm{Au}_{40} \mathrm{~L}_{24}$ & $118(41)$ & $\mathrm{Au}_{41} \mathrm{~L}_{26.5}$ \\
\hline 119(41) & $\mathrm{Au}_{41} \mathrm{~L}_{24.5}$ & $121(42)$ & $\mathrm{Au}_{42} \mathrm{~L}_{27}$ \\
\hline $122(42)$ & $\mathrm{Au}_{42} \mathrm{~L}_{25}$ & $124(43)$ & $\mathrm{Au}_{43} \mathrm{~L}_{27.5}$ \\
\hline $125(43)$ & $\mathrm{Au}_{43} \mathrm{~L}_{25.5}$ & $127(44)$ & $\mathrm{Au}_{44} \mathrm{~L}_{28}$ \\
\hline $128(44)$ & $\mathrm{Au}_{44} \mathrm{~L}_{26}$ & $130(45)$ & $\mathrm{Au}_{45} \mathrm{~L}_{28.5}$ \\
\hline 131(45) & $\mathrm{Au}_{45} \mathrm{~L}_{26.5}$ & $133(46)$ & $\mathrm{Au}_{46} \mathrm{~L}_{29}$ \\
\hline 134(46) & $\mathrm{Au}_{46} \mathrm{~L}_{27}$ & $136(47)$ & $\mathrm{Au}_{47} \mathrm{~L}_{29.5}$ \\
\hline
\end{tabular}


Table 4. Continued

\begin{tabular}{|c|c|c|c|c|c|c|c|}
\hline $\begin{array}{l}\text { HYPOTH } \\
\text { T4 }\end{array}$ & ETICAL & DERIVA & TIONS & & & & \\
\hline$[\mathrm{M}-4]$ & {$\left[\mathrm{AuL}_{3.5}\right]$} & {$[\mathrm{M}-3]$} & {$\left[\mathrm{AuL}_{3.5}\right]$} & {$[\mathrm{M}-2]$} & {$\left[\mathrm{AuL}_{3.5}\right]$} & {$[\mathrm{M}-1]$} & {$\left[\mathrm{AuL}_{3.5}\right]$} \\
\hline K(n) & [M-4] & $K(n)$ & [M-3] & $K(n)$ & [M-2] & $K(n)$ & [M-1] \\
\hline$-9(-4)$ & $-\mathrm{Au}_{4} \mathrm{~L}_{5}$ & $-7(-3)$ & $-\mathrm{Au}_{3} \mathrm{~L}_{3.5}$ & $-5(-2)$ & $-\mathrm{Au}_{2} \mathrm{~L}_{2}$ & $-3(-1)$ & $-\mathrm{AuL}_{0.5}$ \\
\hline$-6(-3)$ & $-\mathrm{Au}_{3} \mathrm{~L}_{4.5}$ & $-4(-2)$ & $-\mathrm{Au}_{2} \mathrm{~L}_{3}$ & $-2(-1)$ & $-\mathrm{AuL}_{1.5}$ & $0(0)$ & 0 \\
\hline$-3(-2)$ & $-\mathrm{Au}_{2} \mathrm{~L}_{4}$ & $-1(-1)$ & $-\mathrm{AuL}_{2.5}$ & $1(0)$ & $-\mathrm{L}$ & $3(1)$ & $\mathrm{AuL}_{0.5}$ \\
\hline $0(-1)$ & $-\mathrm{Au}_{1} \mathrm{~L}_{3.5}$ & $2(0)$ & $-\mathrm{L}_{2}$ & $4(1)$ & $\mathrm{Au}_{1} \mathrm{~L}-0.5$ & $6(2)$ & $\mathrm{Au}_{2} \mathrm{~L}$ \\
\hline $3(0)$ & $-\mathrm{L}_{3}$ & $5(1)$ & $\mathrm{AuL}_{-1.5}$ & $7(2)$ & $\mathbf{A u} \mathbf{u}_{2}$ & $9(3)$ & $\mathrm{Au}_{3} \mathrm{~L}_{1.5}$ \\
\hline $6(1)$ & $\mathrm{AuL}-2.5$ & $8(2)$ & $\mathrm{Au}_{2} \mathrm{~L}_{-1}$ & $10(3)$ & $\mathrm{Au}_{3} \mathrm{~L}_{0.5}$ & $12(4)$ & $\mathrm{Au}_{4} \mathrm{~L}_{2}$ \\
\hline $9(2)$ & $\mathrm{Au}_{2} \mathrm{~L}_{-2}$ & 11(3) & $\mathrm{Au}_{3} \mathrm{~L}_{-0.5}$ & $13(4)$ & $\mathrm{Au}_{4} \mathrm{~L}$ & $15(5)$ & $\mathrm{Au}_{5} \mathrm{~L}_{2.5}$ \\
\hline $12(3)$ & $\mathrm{Au}_{3} \mathrm{~L}_{-1.5}$ & $14(4)$ & $\mathbf{A} \mathbf{u}_{4}$ & $16(5)$ & $\mathrm{Au}_{5} \mathrm{~L}_{1.5}$ & $18(6)$ & $\mathrm{Au}_{6} \mathrm{~L}_{3}$ \\
\hline $15(4)$ & $\mathrm{Au}_{4} \mathrm{~L}_{-1}$ & $17(5)$ & $\mathrm{Au}_{5} \mathrm{~L}_{0.5}$ & $19(6)$ & $\mathrm{Au}_{6} \mathrm{~L}_{2}$ & $21(7)$ & $\mathrm{Au}_{7} \mathrm{~L}_{3.5}$ \\
\hline $18(5)$ & $\mathrm{Au}_{5} \mathrm{~L}_{-0.5}$ & $20(6)$ & $\mathrm{Au}_{6} \mathrm{~L}_{1}$ & $22(7)$ & $\mathrm{Au}_{7} \mathrm{~L}_{2.5}$ & $24(8)$ & $\mathrm{Au}_{8} \mathrm{~L}_{4}$ \\
\hline $21(6)$ & $\mathbf{A u} u_{6}$ & $23(7)$ & $\mathrm{Au}_{7} \mathrm{~L}_{1.5}$ & $25(8)$ & $\mathrm{Au}_{8} \mathrm{~L}_{3}$ & $27(9)$ & $\mathrm{Au}_{9} \mathrm{~L}_{4.5}$ \\
\hline $24(7)$ & $\mathrm{Au}_{7} \mathrm{~L}_{0.5}$ & $26(8)$ & $\mathrm{Au}_{8} \mathrm{~L}_{2}$ & $28(9)$ & $\mathrm{Au}_{9} \mathrm{~L}_{3.5}$ & $30(10)$ & $\mathrm{Au}_{10} \mathrm{~L}_{5}$ \\
\hline $27(8)$ & $\mathrm{Au}_{8} \mathrm{~L}_{1}$ & $29(9)$ & $\mathrm{Au}_{9} \mathrm{~L}_{2.5}$ & $31(10)$ & $\mathrm{Au}_{10} \mathrm{~L}_{4}$ & $33(11)$ & $\mathrm{Au}_{11} \mathrm{~L}_{5.5}$ \\
\hline $30(9)$ & $\mathrm{Au}_{9} \mathrm{~L}_{1.5}$ & $32(10)$ & $\mathrm{Au}_{10} \mathrm{~L}_{3}$ & $34(11)$ & $\mathrm{Au}_{11} \mathrm{~L}_{4.5}$ & $36(12)$ & $\mathrm{Au}_{12} \mathrm{~L}_{6}$ \\
\hline $33(10)$ & $\mathrm{Au}_{10} \mathrm{~L}_{2}$ & $35(11)$ & $\mathrm{Au}_{11} \mathrm{~L}_{3.5}$ & $37(12)$ & $\mathrm{Au}_{12} \mathrm{~L}_{5}$ & $39(13)$ & $\mathrm{Au}_{13} \mathrm{~L}_{6.5}$ \\
\hline $36(11)$ & $\mathrm{Au}_{11} \mathrm{~L}_{2.5}$ & $38(12)$ & $\mathrm{Au}_{12} \mathrm{~L}_{4}$ & $40(13)$ & $\mathrm{Au}_{13} \mathrm{~L}_{5.5}$ & $42(14)$ & $\mathrm{Au}_{14} \mathrm{~L}_{7}$ \\
\hline $39(12)$ & $\mathrm{Au}_{12} \mathrm{~L}_{3}$ & 41(13) & $\mathrm{Au}_{13} \mathrm{~L}_{4.5}$ & $43(14)$ & $\mathrm{Au}_{14} \mathrm{~L}_{6}$ & $45(15)$ & $\mathrm{Au}_{15} \mathrm{~L}_{7.5}$ \\
\hline $42(13)$ & $\mathrm{Au}_{13} \mathrm{~L}_{3.5}$ & $44(14)$ & $\mathrm{Au}_{14} \mathrm{~L}_{5}$ & $46(15)$ & $\mathrm{Au}_{15} \mathrm{~L}_{6.5}$ & $48(16)$ & $\mathrm{Au}_{16} \mathrm{~L}_{8}$ \\
\hline $45(14)$ & $\mathrm{Au}_{14} \mathrm{~L}_{4}$ & $47(15)$ & $\mathrm{Au}_{15} \mathrm{~L}_{5.5}$ & $49(16)$ & $\mathrm{Au}_{16} \mathrm{~L}_{7}$ & $51(17)$ & $\mathrm{Au}_{17} \mathrm{~L}_{8.5}$ \\
\hline $48(15)$ & $\mathrm{Au}_{15} \mathrm{~L}_{4.5}$ & $50(16)$ & $\mathrm{Au}_{16} \mathrm{~L}_{6}$ & $52(17)$ & $\mathrm{Au}_{17} \mathrm{~L}_{7.5}$ & $54(18)$ & $\mathrm{Au}_{18} \mathrm{~L}_{9}$ \\
\hline $51(16)$ & $\mathrm{Au}_{16} \mathrm{~L}_{5}$ & $53(17)$ & $\mathrm{Au}_{17} \mathrm{~L}_{6.5}$ & $55(18)$ & $\mathrm{Au}_{18} \mathrm{~L}_{8}$ & $57(19)$ & $\mathrm{Au}_{19} \mathrm{~L}_{9.5}$ \\
\hline $54(17)$ & $\mathrm{Au}_{17} \mathrm{~L}_{5.5}$ & $56(18)$ & $\mathrm{Au}_{18} \mathrm{~L}_{7}$ & $58(19)$ & $\mathrm{Au}_{19} \mathrm{~L}_{8.5}$ & $60(20)$ & $\mathrm{Au}_{20} \mathrm{~L}_{10}$ \\
\hline $57(18)$ & $\mathrm{Au}_{18} \mathrm{~L}_{6}$ & $59(19)$ & $\mathrm{Au}_{19} \mathrm{~L}_{7.5}$ & $61(20)$ & $\mathrm{Au}_{20} \mathrm{~L}_{9}$ & $63(21)$ & $\mathrm{Au}_{21} \mathrm{~L}_{10.5}$ \\
\hline $60(19)$ & $\mathrm{Au}_{19} \mathrm{~L}_{6.5}$ & $62(20)$ & $\mathrm{Au}_{20} \mathrm{~L}_{8}$ & $64(21)$ & $\mathrm{Au}_{21} \mathrm{~L}_{9.5}$ & $66(22)$ & $\mathrm{Au}_{22} \mathrm{~L}_{11}$ \\
\hline $63(20)$ & $\mathrm{Au}_{20} \mathrm{~L}_{7}$ & $65(21)$ & $\mathrm{Au}_{21} \mathrm{~L}_{8.5}$ & $67(22)$ & $\mathrm{Au}_{22} \mathrm{~L}_{10}$ & $69(23)$ & $\mathrm{Au}_{23} \mathrm{~L}_{11.5}$ \\
\hline $66(21)$ & $\mathrm{Au}_{21} \mathrm{~L}_{7.5}$ & $68(22)$ & $\mathrm{Au}_{22} \mathrm{~L}_{9}$ & $70(23)$ & $\mathrm{Au}_{23} \mathrm{~L}_{10.5}$ & $72(24)$ & $\mathrm{Au}_{24} \mathrm{~L}_{12}$ \\
\hline $69(22)$ & $\mathrm{Au}_{22} \mathrm{~L}_{8}$ & $71(23)$ & $\mathrm{Au}_{23} \mathrm{~L}_{9.5}$ & $73(24)$ & $\mathrm{Au}_{24} \mathrm{~L}_{11}$ & $75(25)$ & $\mathrm{Au}_{25} \mathrm{~L}_{12.5}$ \\
\hline $72(23)$ & $\mathrm{Au}_{23} \mathrm{~L}_{8.5}$ & $74(24)$ & $\mathrm{Au}_{24} \mathrm{~L}_{10}$ & $76(25)$ & $\mathrm{Au}_{25} \mathrm{~L}_{11.5}$ & $78(26)$ & $\mathrm{Au}_{26} \mathrm{~L}_{13}$ \\
\hline $75(24)$ & $\mathrm{Au}_{24} \mathrm{~L}_{9}$ & $77(25)$ & $\mathrm{Au}_{25} \mathrm{~L}_{10.5}$ & $79(26)$ & $\mathrm{Au}_{26} \mathrm{~L}_{12}$ & $81(27)$ & $\mathrm{Au}_{27} \mathrm{~L}_{13.5}$ \\
\hline $78(25)$ & $\mathbf{A} \mathbf{u}_{25} \mathbf{L}_{9.5}$ & $80(26)$ & $\mathrm{Au}_{26} \mathrm{~L}_{11}$ & $82(27)$ & $\mathrm{Au}_{27} \mathrm{~L}_{12.5}$ & $84(28)$ & $\mathrm{Au}_{28} \mathrm{~L}_{14}$ \\
\hline $81(26)$ & $\mathrm{Au}_{26} \mathrm{~L}_{10}$ & $83(27)$ & $\mathrm{Au}_{27} \mathrm{~L}_{11.5}$ & $85(28)$ & $\mathrm{Au}_{28} \mathrm{~L}_{13}$ & $87(29)$ & $\mathrm{Au}_{29} \mathrm{~L}_{14.5}$ \\
\hline $84(27)$ & $\mathrm{Au}_{27} \mathrm{~L}_{10.5}$ & $86(28)$ & $\mathrm{Au}_{28} \mathrm{~L}_{12}$ & $88(29)$ & $\mathrm{Au}_{29} \mathrm{~L}_{13.5}$ & $90(30)$ & $\mathrm{Au}_{30} \mathrm{~L}_{15}$ \\
\hline $87(28)$ & $\mathrm{Au}_{28} \mathrm{~L}_{11}$ & $89(29)$ & $\mathrm{Au}_{29} \mathrm{~L}_{12.5}$ & $91(30)$ & $\mathrm{Au}_{30} \mathrm{~L}_{14}$ & $93(31)$ & $\mathrm{Au}_{31} \mathrm{~L}_{15.5}$ \\
\hline $90(29)$ & $\mathrm{Au}_{29} \mathrm{~L}_{11.5}$ & $92(30)$ & $\mathrm{Au}_{30} \mathrm{~L}_{13}$ & $94(31)$ & $\mathrm{Au}_{31} \mathrm{~L}_{14.5}$ & $96(32)$ & $\mathrm{Au}_{32} \mathrm{~L}_{16}$ \\
\hline $93(30)$ & $\mathrm{Au}_{30} \mathrm{~L}_{12}$ & $95(31)$ & $\mathrm{Au}_{31} \mathrm{~L}_{13.5}$ & $97(32)$ & $\mathrm{Au}_{32} \mathrm{~L}_{15}$ & $99(33)$ & $\mathrm{Au}_{33} \mathrm{~L}_{16.5}$ \\
\hline $96(31)$ & $\mathrm{Au}_{31} \mathrm{~L}_{12.5}$ & $98(32)$ & $\mathrm{Au}_{32} \mathrm{~L}_{14}$ & $100(33)$ & $\mathrm{Au}_{33} \mathrm{~L}_{15.5}$ & $102(34)$ & $\mathrm{Au}_{34} \mathrm{~L}_{17}$ \\
\hline $99(32)$ & $\mathrm{Au}_{32} \mathrm{~L}_{13}$ & 101(33) & $\mathrm{Au}_{33} \mathrm{~L}_{14.5}$ & $103(34)$ & $\mathrm{Au}_{34} \mathrm{~L}_{16}$ & $105(35)$ & $\mathrm{Au}_{35} \mathrm{~L}_{17.5}$ \\
\hline $102(33)$ & $\mathrm{Au}_{33} \mathrm{~L}_{13.5}$ & $104(34)$ & $\mathrm{Au}_{34} \mathrm{~L}_{15}$ & $106(35)$ & $\mathrm{Au}_{35} \mathrm{~L}_{16.5}$ & $108(36)$ & $\mathrm{Au}_{36} \mathrm{~L}_{18}$ \\
\hline $105(34)$ & $\mathrm{Au}_{34} \mathrm{~L}_{14}$ & $107(35)$ & $\mathrm{Au}_{35} \mathrm{~L}_{15.5}$ & $109(36)$ & $\mathrm{Au}_{36} \mathrm{~L}_{17}$ & $111(37)$ & $\mathrm{Au}_{37} \mathrm{~L}_{18.5}$ \\
\hline $108(35)$ & $\mathrm{Au}_{35} \mathrm{~L}_{14.5}$ & $110(36)$ & $\mathrm{Au}_{36} \mathrm{~L}_{16}$ & $112(37)$ & $\mathrm{Au}_{37} \mathrm{~L}_{17.5}$ & $114(38)$ & $\mathrm{Au}_{38} \mathrm{~L}_{19}$ \\
\hline $111(36)$ & $\mathrm{Au}_{36} \mathrm{~L}_{15}$ & $113(37)$ & $\mathrm{Au}_{37} \mathrm{~L}_{16.5}$ & $115(38)$ & $\mathrm{Au}_{38} \mathrm{~L}_{18}$ & $117(39)$ & $\mathrm{Au}_{39} \mathrm{~L}_{19.5}$ \\
\hline $114(37)$ & $\mathrm{Au}_{37} \mathrm{~L}_{15.5}$ & $116(38)$ & $\mathrm{Au}_{38} \mathrm{~L}_{17}$ & $118(39)$ & $\mathrm{Au}_{39} \mathrm{~L}_{18.5}$ & $120(40)$ & $\mathrm{Au}_{40} \mathrm{~L}_{20}$ \\
\hline
\end{tabular}


Table 5. Selected known Golden

\begin{tabular}{|c|c|c|c|c|c|}
\hline $\begin{array}{l}\text { T5 } \\
\text { EXAMPLES } \\
\text { CATEGORIZE }\end{array}$ & CLUSTER & $\begin{array}{l}\text { GROUPS } \\
\mathrm{K}(\mathrm{n})\end{array}$ & $\begin{array}{l}\mathrm{K}(\mathrm{n}) \text { Multiples } \\
{\left[\mathrm{AuL}_{3.5}\right]}\end{array}$ & SERIES & $\mathrm{Kp}$ \\
\hline $\mathrm{Au}_{144} \mathrm{R}_{60}$ & $\mathrm{Au}_{144} \mathrm{~L}_{30}$ & $474(144)$ & $144\left[\mathrm{AuL}_{3.5}\right]-474 \mathrm{~L}=\mathrm{Au}_{144} \mathrm{~L}_{30}$ & $4 n-372$ & $\mathrm{C}^{187} \mathrm{C}[\mathrm{M}-43]$ \\
\hline $\mathrm{Au}_{102} \mathrm{R}_{44}$ & $\mathrm{Au}_{102} \mathrm{~L}_{22}$ & $335(102)$ & $102\left[\mathrm{AuL}_{3.5}\right]-335 \mathrm{~L}=\mathrm{Au}_{102} \mathrm{~L}_{22}$ & $4 n-262$ & $\mathrm{C}^{132} \mathrm{C}[\mathrm{M}-30]$ \\
\hline $\mathrm{Au}_{69} \mathrm{~L}_{20} \mathrm{Cl}_{12}-$ & $\mathrm{Au}_{69} \mathrm{~L}_{26.5}$ & $215(69)$ & $69\left[\mathrm{AuL}_{3.5}\right]-215 \mathrm{~L}=\mathrm{Au}_{69} \mathrm{~L}_{26.5}$ & $4 n-154$ & $\mathrm{C}^{78} \mathrm{C}[\mathrm{M}-9]$ \\
\hline $\mathrm{Au}_{55} \mathrm{~L}_{12} \mathrm{Cl}_{6}^{+}$ & $\mathrm{Au}_{55} \mathrm{~L}_{14.5}$ & $178(55)$ & $55\left[\mathrm{AuL}_{3.5}\right]-178 \mathrm{~L}=\mathrm{Au}_{55} \mathrm{~L}_{14.5}$ & $4 n-136$ & $\mathrm{C}^{69} \mathrm{C}[\mathrm{M}-14]$ \\
\hline $\mathrm{Au}_{55} \mathrm{~L}_{12} \mathrm{Cl}_{6}^{-}$ & $\mathrm{Au}_{55} \mathrm{~L}_{15.5}$ & $177(55)$ & $55\left[\mathrm{AuL}_{3.5}\right]-177 \mathrm{~L}=\mathrm{Au}_{55} \mathrm{~L}_{15.5}$ & $4 n-134$ & $\mathrm{C}^{68} \mathrm{C}[\mathrm{M}-13]$ \\
\hline $\mathrm{Au}_{38} \mathrm{R}_{24}$ & $\mathrm{Au}_{38} \mathrm{~L}_{12}$ & $121(38)$ & $38\left[\mathrm{AuL}_{3.5}\right]-121 \mathrm{~L}=\mathrm{Au}_{38} \mathrm{~L}_{12}$ & $4 n-90$ & $\mathrm{C}^{46} \mathrm{C}[\mathrm{M}-8]$ \\
\hline $\mathrm{Au}_{36} \mathrm{R}_{24}$ & $\mathrm{Au}_{36} \mathrm{~L}_{12}$ & $114(36)$ & $36\left[\mathrm{AuL}_{3.5}\right]-114 \mathrm{~L}=\mathrm{Au}_{36} \mathrm{~L}_{12}$ & $4 n-86$ & $\mathrm{C}^{43} \mathrm{C}[\mathrm{M}-7]$ \\
\hline $\mathrm{Au}_{39} \mathrm{~L}_{14} \mathrm{Cl}_{6}{ }^{3+}$ & $\mathrm{Au}_{39} \mathrm{~L}_{15.5}$ & $121(39)$ & $36\left[\mathrm{AuL}_{3.5}\right]-114 \mathrm{~L}=\mathrm{Au}_{36} \mathrm{~L}_{12}$ & $4 n-86$ & $\mathrm{C}^{44} \mathrm{C}[\mathrm{M}-5]$ \\
\hline $\mathrm{Au}_{25} \mathrm{R}_{18}^{+}$ & $\mathrm{Au}_{25} \mathrm{~L}_{8.5}$ & $79(25)$ & $25\left[\mathrm{AuL}_{3.5}\right]-79 \mathrm{~L}=\mathrm{Au}_{25} \mathrm{~L}_{8.5}$ & $4 n-58$ & $\mathrm{C}^{30} \mathrm{C}[\mathrm{M}-5]$ \\
\hline $\mathrm{Au}_{25} \mathbf{R}_{18}-$ & $\mathrm{Au}_{25} \mathbf{L}_{9.5}$ & $78(25)$ & $25\left[\mathrm{AuL}_{3.5}\right]-78 \mathrm{~L}=\mathrm{Au}_{25} \mathrm{~L}_{9.5}$ & $4 n-56$ & $\mathrm{C}^{29} \mathrm{C}[\mathrm{M}-4]$ \\
\hline $\mathrm{Au}_{18} \mathrm{Ag}_{19} \mathrm{Br}_{11} \mathrm{~L}_{12}{ }^{2+}$ & $\mathrm{Au}_{37} \mathrm{~L}_{16.5}$ & $113(37)$ & $37\left[\mathrm{AuL}_{3.5}\right]-113 \mathrm{~L}=\mathrm{Au}_{37} \mathrm{~L}_{16.5}$ & $4 n-78$ & $\mathrm{C}^{40} \mathrm{C}[\mathrm{M}-3]$ \\
\hline $\mathrm{Au}_{38} \mathrm{~L}_{18} \mathrm{Cl}_{2}^{4+}$ & $\mathrm{Au}_{38} \mathrm{~L}_{17}$ & $116(38)$ & $38\left[\mathrm{AuL}_{3.5}\right]-116 \mathrm{~L}=\mathrm{Au}_{38} \mathrm{~L}_{17}$ & $4 n-80$ & $\mathrm{C}^{41} \mathrm{C}[\mathrm{M}-3]$ \\
\hline $\mathrm{Au}_{25} \mathrm{~L}_{10} \mathrm{R}_{5}{ }^{2+}$ & $\mathrm{Au}_{25} \mathrm{~L}_{11.5}$ & $76(25)$ & $25\left[\mathrm{AuL}_{3.5}\right]-76 \mathrm{~L}=\mathrm{Au}_{25} \mathrm{~L}_{11.5}$ & $4 n-52$ & $\mathrm{C}^{27} \mathrm{C}[\mathrm{M}-2]$ \\
\hline $\mathrm{Au}_{68} \mathrm{R}_{34}$ & $\mathrm{Au}_{68} \mathrm{~L}_{17}$ & $221(68)$ & $68\left[\mathrm{AuL}_{3.5}\right]-221 \mathrm{~L}=\mathrm{Au}_{68} \mathrm{~L}_{17}$ & $4 n-136$ & $\mathrm{C}^{69} \mathrm{C}[\mathrm{M}-1]$ \\
\hline $\mathrm{Au}_{24} \mathrm{~L}_{10} \mathrm{R}_{5}^{+}$ & $\mathrm{Au}_{24} \mathrm{~L}_{12}$ & $72(24)$ & $24\left[\mathrm{AuL}_{3.5}\right]-72 \mathrm{~L}=\mathrm{Au}_{24} \mathrm{~L}_{12}$ & $4 n-48$ & $\mathrm{C}^{25} \mathrm{C}[\mathrm{M}-1]$ \\
\hline $\mathrm{Au}_{10} \mathrm{~L}_{3} \mathrm{R}_{4}$ & $\mathrm{Au}_{10} \mathrm{~L}_{5}$ & $30(10)$ & $10\left[\mathrm{AuL}_{3.5}\right]-30 \mathrm{~L}=\mathrm{Au}_{10} \mathrm{~L}_{5}$ & $4 n-20$ & $\mathrm{C}^{11} \mathrm{C}[\mathrm{M}-1]$ \\
\hline $\mathrm{AuL}_{2}^{+}$ & & $2(1)$ & $1\left[\mathrm{AuL}_{3.5}\right]-2 \mathrm{~L}=\mathrm{Au}_{1} \mathrm{~L}_{1.5}$ & $4 n+0$ & $\mathrm{C}^{1} \mathrm{C}[\mathrm{M} 0]$ \\
\hline $\mathrm{AuClL}$ & $\mathrm{AuL}_{1.5}$ & $2(1)$ & $1\left[\mathrm{AuL}_{3.5}\right]-2 \mathrm{~L}=\mathrm{Au}_{1} \mathrm{~L}_{1.5}$ & $4 n+0$ & $\mathrm{C}^{1} \mathrm{C}[\mathrm{M} 0]$ \\
\hline $\mathrm{Au}_{2} \mathrm{~L}_{2}$ & $\mathrm{Au}_{2} \mathrm{~L}_{2}$ & $5(2)$ & $2\left[\mathrm{AuL}_{3.5}\right]-5 \mathrm{~L}=\mathbf{A u}_{\mathbf{2}} \mathbf{L}_{2}$ & $4 n-2$ & $\mathrm{C}^{2} \mathrm{C}[\mathrm{M} 0]$ \\
\hline $\mathrm{Au}_{3} \mathrm{~L}_{3}^{+}$ & $\mathrm{Au}_{3} \mathrm{~L}_{2.5}$ & $8(3)$ & $3\left[\mathrm{AuL}_{3.5}\right]-8 \mathrm{~L}=\mathrm{Au}_{3} \mathrm{~L}_{2.5}$ & $4 n-4$ & $\mathrm{C}^{3} \mathrm{C}[\mathrm{M} 0]$ \\
\hline $\mathrm{Au}_{8} \mathrm{~L}_{6}^{2+}$ & $\mathrm{Au}_{8} \mathrm{~L}_{5}$ & $23(8)$ & $8\left[\mathrm{AuL}_{3.5}\right]-23 \mathrm{~L}=\mathrm{Au}_{8} \mathrm{~L}_{5}$ & $4 n-14$ & $\mathrm{C}^{8} \mathrm{C}[\mathrm{M} 0]$ \\
\hline $\mathrm{Au}_{20} \mathrm{~L}_{10} \mathrm{Cl}_{4}{ }^{2+}$ & $\mathrm{Au}_{20} \mathrm{~L}_{12}$ & $59(20)$ & $20\left[\mathrm{AuL}_{3.5}\right]-59 \mathrm{~L}=\mathrm{Au}_{20} \mathrm{~L}_{12}$ & $4 n-38$ & $\mathrm{C}^{20} \mathrm{C}[\mathrm{M} 0]$ \\
\hline $\mathrm{Au}_{22} \mathrm{~L}_{12}$ & $\mathrm{Au}_{22} \mathrm{~L}_{12}$ & $65(22)$ & $22\left[\mathrm{AuL}_{3.5}\right]-65 \mathrm{~L}=\mathrm{Au}_{22} \mathrm{~L}_{12}$ & $4 n-42$ & $\mathrm{C}^{22} \mathrm{C}[\mathrm{M} 0]$ \\
\hline $\mathrm{Au}_{24} \mathrm{~L}_{10} \mathrm{R}_{5} \mathrm{X}_{2}^{+}$ & $\mathrm{Au}_{24} \mathrm{~L}_{13}$ & $71(24)$ & $24\left[\mathrm{AuL}_{3.5}\right]-71 \mathrm{~L}=\mathrm{Au}_{24} \mathrm{~L}_{13}$ & $4 n-46$ & $\mathrm{C}^{24} \mathrm{C}[\mathrm{M} 0]$ \\
\hline $\mathrm{Au}_{2} \mathrm{~L}_{2} \mathrm{Cl}_{2}$ & $\mathrm{Au}_{2} \mathrm{~L}_{3}$ & $4(2)$ & $2\left[\mathrm{AuL}_{3.5}\right]-4 \mathrm{~L}=\mathrm{Au}_{2} \mathrm{~L}_{3}$ & $4 n+0$ & $\mathrm{C}^{1} \mathrm{C}[\mathrm{M} 1]$ \\
\hline $\mathrm{Au}_{4} \mathrm{~L}_{4}$ & $\mathrm{Au}_{4} \mathrm{~L}_{4}$ & $10(4)$ & $4\left[\mathrm{AuL}_{3.5}\right]-10 \mathrm{~L}=\mathrm{Au}_{4} \mathrm{~L}_{4}$ & $4 n-4$ & $\mathrm{C}^{3} \mathrm{C}[\mathrm{M} 1]$ \\
\hline $\mathrm{Au}_{5} \mathrm{~L}_{4} \mathrm{Cl}$ & $\mathrm{Au}_{5} \mathrm{~L}_{4.5}$ & $13(5)$ & $5\left[\mathrm{AuL}_{3.5}\right]-13 \mathrm{~L}=\mathrm{Au}_{5} \mathrm{~L}_{4.5}$ & $4 n-6$ & $\mathrm{C}^{4} \mathrm{C}[\mathrm{M} 1]$ \\
\hline $\mathrm{Au}_{6} \mathrm{~L}_{4} \mathrm{Cl}_{2}$ & $\mathrm{Au}_{6} \mathrm{~L}_{5}$ & $16(6)$ & $6\left[\mathrm{AuL}_{3.5}\right]-16 \mathrm{~L}=\mathrm{Au}_{6} \mathrm{~L}_{5}$ & $4 n-8$ & $\mathrm{C}^{5} \mathrm{C}[\mathrm{M} 1]$ \\
\hline $\mathrm{Au}_{6} \mathrm{~L}_{6}^{2+}$ & $\mathrm{Au}_{6} \mathrm{~L}_{5}$ & $16(6)$ & $6\left[\mathrm{AuL}_{3.5}\right]-16 \mathrm{~L}=\mathrm{Au}_{6} \mathrm{~L}_{5}$ & $4 n-8$ & $\mathrm{C}^{5} \mathrm{C}[\mathrm{M} 1]$ \\
\hline $\mathrm{Au}_{8} \mathrm{~L}_{7}^{2+}$ & $\mathrm{Au}_{8} \mathrm{~L}_{6}$ & $22(8)$ & $8\left[\mathrm{AuL}_{3.5}\right]-22 \mathrm{~L}=\mathrm{Au}_{8} \mathrm{~L}_{6}$ & $4 n-12$ & $\mathrm{C}^{7} \mathrm{C}[\mathrm{M} 1]$ \\
\hline $\mathrm{Au}_{9} \mathrm{~L}_{8}{ }^{3+}$ & $\mathrm{Au}_{9} \mathrm{~L}_{6.5}$ & $25(9)$ & $9\left[\mathrm{AuL}_{3.5}\right]-25 \mathrm{~L}=\mathrm{Au}_{9} \mathrm{~L}_{6.5}$ & $4 n-14$ & $\mathrm{C}^{8} \mathrm{C}[\mathrm{M} 1]$ \\
\hline $\mathrm{Au}_{9} \mathrm{~L}_{5} \mathrm{R}_{3}$ & $\mathrm{Au}_{9} \mathrm{~L}_{6.5}$ & $25(9)$ & $9\left[\mathrm{AuL}_{3.5}\right]-25 \mathrm{~L}=\mathrm{Au}_{9} \mathrm{~L}_{6.5}$ & $4 n-14$ & $\mathrm{C}^{8} \mathrm{C}[\mathrm{M} 1]$ \\
\hline $\mathrm{Au}_{10} \mathrm{~L}_{6} \mathrm{Cl}_{3}^{+}$ & $\mathrm{Au}_{10} \mathrm{~L}_{7}$ & $28(10)$ & $10\left[\mathrm{AuL}_{3.5}\right]-28 \mathrm{~L}=\mathrm{Au}_{10} \mathrm{~L}_{7}$ & $4 n-16$ & $\mathrm{C}^{9} \mathrm{C}[\mathrm{M} 1]$ \\
\hline $\mathrm{AuL}_{4}^{+}$ & $0(1)$ & $1\left[\mathrm{AuL}{ }_{3.5}\right]$ & $1\left[\mathrm{AuL}_{3.5}\right]-0 \mathrm{~L}=\mathrm{Au}_{1} \mathrm{~L}_{3.5}$ & $4 n+4$ & $\mathrm{C}^{-1} \mathrm{C}[\mathrm{M} 2]$ \\
\hline $\mathrm{Au}_{4} \mathrm{~L}_{4} \mathrm{I}_{2}$ & $\mathrm{Au}_{4} \mathrm{~L}_{5}$ & $9(4)$ & $4\left[\mathrm{AuL}_{3.5}\right]-9 \mathrm{~L}=\mathrm{Au}_{4} \mathrm{~L}_{5}$ & $4 n-2$ & $\mathrm{C}^{2} \mathrm{C}[\mathrm{M} 2]$ \\
\hline $\mathrm{Au}_{7} \mathrm{~L}_{7}^{+}$ & $\mathrm{Au}_{7} \mathrm{~L}_{6.5}$ & $18(7)$ & $7\left[\mathrm{AuL}_{3.5}\right]-18 \mathrm{~L}=\mathrm{Au}_{7} \mathrm{~L}_{6.5}$ & $4 n-8$ & $\mathrm{C}^{5} \mathrm{C}[\mathrm{M} 2]$ \\
\hline $\mathrm{Au}_{8} \mathrm{~L}_{8}{ }^{2+}$ & $\mathrm{Au}_{8} \mathrm{~L}_{7}$ & $21(8)$ & $8\left[\mathrm{AuL}_{3.5}\right]-21 \mathrm{~L}=\mathrm{Au}_{8} \mathrm{~L}_{7}$ & $4 n-10$ & $\mathrm{C}^{6} \mathrm{C}[\mathrm{M} 2]$ \\
\hline $\mathrm{Au}_{9} \mathrm{~L}_{8}^{+}$ & $\mathrm{Au}_{9} \mathrm{~L}_{7.5}$ & 24(9) & $9\left[\mathrm{AuL}_{3.5}\right]-24 \mathrm{~L}=\mathrm{Au}_{9} \mathrm{~L}_{7.5}$ & $4 n-12$ & $\mathrm{C}^{7} \mathrm{C}[\mathrm{M} 2]$ \\
\hline CLUSTER & CLUSTER & $\mathrm{K}(\mathrm{n})$ & K(n) Multiples & SERIES & $\mathrm{Kp}$ \\
\hline $\mathrm{Au}_{11} \mathrm{~L}_{7} \mathrm{X}_{3}$ & $\mathrm{Au}_{11} \mathrm{~L}_{8.5}$ & $30(11)$ & $11\left[\mathrm{AuL}_{3.5}\right]-30 \mathrm{~L}=\mathrm{Au}_{11} \mathrm{~L}_{8.5}$ & $4 n-16$ & $\mathrm{C}^{9} \mathrm{C}[\mathrm{M} 2]$ \\
\hline $\mathrm{Au}_{11} \mathrm{~L}_{8} \mathrm{Cl}_{2}^{+}$ & $\mathrm{Au}_{11} \mathrm{~L}_{8.5}$ & $30(11)$ & $11\left[\mathrm{AuL}_{3.5}\right]-30 \mathrm{~L}=\mathrm{Au}_{11} \mathrm{~L}_{8.5}$ & $4 n-16$ & $\mathrm{C}^{9} \mathrm{C}[\mathrm{M} 2]$ \\
\hline $\mathrm{Au}_{11} \mathrm{I}_{3} \mathrm{~L}_{7}$ & $\mathrm{Au}_{11} \mathrm{~L}_{8.5}$ & $30(11)$ & $11\left[\mathrm{AuL}_{3.5}\right]-30 \mathrm{~L}=\mathrm{Au}_{11} \mathrm{~L}_{8.5}$ & $4 n-16$ & $\mathrm{C}^{9} \mathrm{C}[\mathrm{M} 2]$ \\
\hline $\mathrm{Au}_{11} \mathrm{~L}_{10}{ }^{3+}$ & $\mathrm{Au}_{11} \mathrm{~L}_{8.5}$ & $30(11)$ & $11\left[\mathrm{AuL}_{3.5}\right]-30 \mathrm{~L}=\mathrm{Au}_{11} \mathrm{~L}_{8.5}$ & $4 n-16$ & $\mathrm{C}^{9} \mathrm{C}[\mathrm{M} 2]$ \\
\hline $\mathrm{Au}_{12} \mathrm{~L}_{10} \mathrm{Cl}^{3+}$ & $\mathrm{Au}_{12} \mathrm{~L}_{9}$ & $33(12)$ & $12\left[\mathrm{AuL}_{3.5}\right]-33 \mathrm{~L}=\mathrm{Au}_{12} \mathrm{~L}_{9}$ & $4 n-18$ & $\mathrm{C}^{10} \mathrm{C}[\mathrm{M} 2]$ \\
\hline $\mathrm{Au}_{13} \mathrm{~L}_{8} \mathrm{Cl}_{4}^{+}$ & $\mathrm{Au}_{13} \mathrm{~L}_{9.5}$ & $36(13)$ & $13\left[\mathrm{AuL}_{3.5}\right]-36 \mathrm{~L}=\mathrm{Au}_{13} \mathrm{~L}_{9.5}$ & $4 n-20$ & $\mathrm{C}^{11} \mathrm{C}[\mathrm{M} 2]$ \\
\hline $\mathrm{Au}_{13} \mathrm{~L}_{10} \mathrm{Cl}_{2}^{3+}$ & $\mathrm{Au}_{13} \mathrm{~L}_{9.5}$ & $36(13)$ & $13\left[\mathrm{AuL}_{3.5}\right]-36 \mathrm{~L}=\mathrm{Au}_{13} \mathrm{~L}_{9.5}$ & $4 n-20$ & $\mathrm{C}^{11} \mathrm{C}[\mathrm{M} 2]$ \\
\hline $\mathrm{Au}_{14} \mathrm{~L}_{8}\left(\mathrm{NO}_{3}\right)_{4}$ & $\mathrm{Au}_{14} \mathrm{~L}_{10}$ & $39(14)$ & $14\left[\mathrm{AuL}_{3.5}\right]-39 \mathrm{~L}=\mathrm{Au}_{14} \mathrm{~L}_{10}$ & $4 n-22$ & $\mathrm{C}^{12} \mathrm{C}[\mathrm{M} 2]$ \\
\hline $\mathrm{Au}_{6} \mathrm{~L}_{8}{ }^{2+}$ & $\mathrm{Au}_{6} \mathrm{~L}_{7}$ & $14(6)$ & $6\left[\mathrm{AuL}_{3.5}\right]-14 \mathrm{~L}=\mathrm{Au}_{6} \mathrm{~L}_{7}$ & $4 n-4$ & $\mathrm{C}^{3} \mathrm{C}[\mathrm{M} 3]$ \\
\hline $\mathrm{Au}_{8} \mathrm{~L}_{8} \mathrm{Cl}_{2}^{2+}$ & $\mathrm{Au}_{8} \mathrm{~L}_{8}$ & $20(8)$ & $8\left[\mathrm{AuL}_{3.5}\right]-20 \mathrm{~L}=\mathrm{Au}_{8} \mathrm{~L}_{8}$ & $4 n-8$ & $\mathrm{C}^{5} \mathrm{C}[\mathrm{M} 3]$ \\
\hline $\mathrm{Au}_{8} \mathrm{~L}_{8} \mathrm{R}_{2}^{2+}$ & $\mathrm{Au}_{8} \mathrm{~L}_{8}$ & $20(8)$ & $8\left[\mathrm{AuL}_{3.5}\right]-20 \mathrm{~L}=\mathrm{Au}_{8} \mathrm{~L}_{8}$ & $4 n-8$ & $\mathrm{C}^{5} \mathrm{C}[\mathrm{M} 3]$ \\
\hline $\mathrm{Au}_{15} \mathrm{~L}_{12} \mathrm{Cl}(\mathrm{H})^{3+}$ & $\mathrm{Au}_{15} \mathrm{~L}_{11.5}$ & $41(15)$ & $15\left[\mathrm{AuL}_{3.5}\right]-41 \mathrm{~L}=\mathrm{Au}_{15} \mathrm{~L}_{11.5}$ & $4 n-22$ & $\mathrm{C}^{12} \mathrm{C}[\mathrm{M} 3]$ \\
\hline $\mathrm{Au}_{16} \mathrm{~L}_{8} \mathrm{X}_{8}$ & $\mathrm{Au}_{16} \mathrm{~L}_{12}$ & $44(16)$ & $16\left[\mathrm{AuL}_{3.5}\right]-44 \mathrm{~L}=\mathrm{Au}_{16} \mathrm{~L}_{12}$ & $4 n-24$ & $\mathrm{C}^{13} \mathrm{C}[\mathrm{M} 3]$ \\
\hline $\mathrm{Au}_{8} \mathrm{~L}_{8} \mathrm{X}_{2}$ & $\mathrm{Au}_{8} \mathrm{~L}_{9}$ & $19(8)$ & $8\left[\mathrm{AuL}_{3.5}\right]-19 \mathrm{~L}=\mathrm{Au}_{8} \mathrm{~L}_{9}$ & $4 n-6$ & $\mathrm{C}^{4} \mathrm{C}[\mathrm{M} 4]$ \\
\hline $\mathrm{Au}_{11} \mathrm{~L}_{12}{ }^{3+}$ & $\mathrm{Au}_{11} \mathrm{~L}_{10.5}$ & $28(11)$ & $11\left[\mathrm{AuL}_{3.5}\right]-28 \mathrm{~L}=\mathrm{Au}_{11} \mathrm{~L}_{10.5}$ & $4 n-12$ & $\mathrm{C}^{7} \mathrm{C}[\mathrm{M} 4]$ \\
\hline
\end{tabular}

\subsection{Some Gold Structures and Symmetries}

The beautiful structures and symmetries of golden clusters have widely been discussed(Mingos, 1984;Konishi, 2014;Kiremire, 2016c). However using the capping symbol $\mathrm{Kp}=\mathrm{C}^{\mathrm{y}} \mathrm{C}[\mathrm{Mx}]$ as a guide, good 2-dimensional sketches can generated. These are shown in Figures F-8 to F-16. 
$\mathrm{Au}_{8} \mathrm{~L}^{2+}: \mathrm{K}=8[3.5]-7+1=22(8) ; \mathrm{S}=4 \mathrm{n}-12, \mathrm{Kp}=\mathrm{C}^{7} \mathrm{C}[\mathrm{M} 1]$

The capping symbol tells us that the cluster has ine skeletal element at the nucleus and is surrounded by 7 capping skeletal elements. A skeletal graph of the cluster is shown in F-

$O=A u \quad O=L$

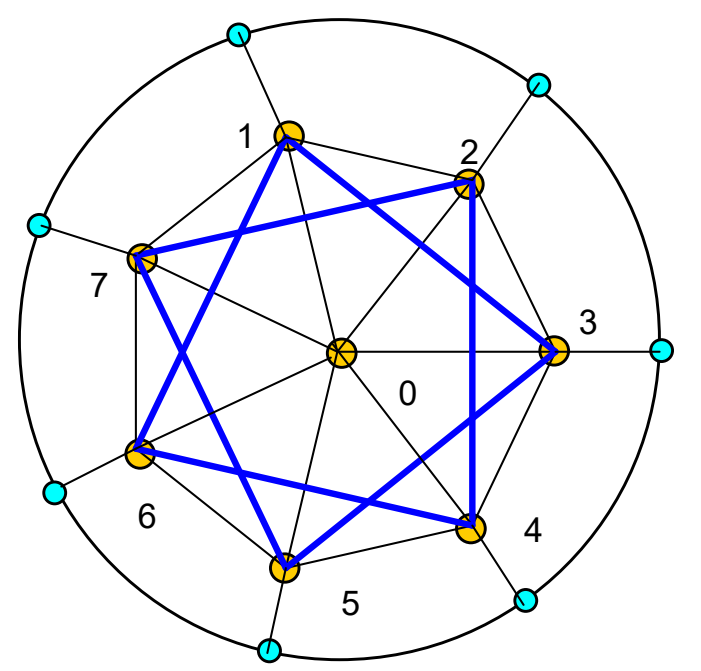

$\mathrm{K} 1=3.5-5(0.5)=1$

$\mathrm{K} 2=\mathrm{K} 1=1$

$\mathrm{K} 3=\mathrm{K} 1=1$

$\mathrm{K} 4=\mathrm{K} 1=1$

$\mathrm{K} 5=\mathrm{K} 1=1$

$\mathrm{K} 6=\mathrm{K} 1=1$

$\mathrm{K} 7=\mathrm{K} 1=1$

$\mathrm{K} 0=1[3.5]-7(0.5)-(1)+2(0.5)=0$

This means that the nucleus has no ligand.

F-8

$\mathrm{Aug}_{8}{ }^{3+}: \mathrm{K}=9[3.5]-8+1.5=25 ; \mathrm{K}(\mathrm{n})=25(9), \mathrm{S}=4 \mathrm{n}-14 ; \mathrm{Kp}=\mathrm{C}^{8} \mathrm{C}[\mathrm{M} 1]$

This capping symbol means that the cluster has one skeletal element acting

as a NUCLEUS which is surrounded by 8 capping skeletal elements. This is represented by a graphical sketch in F-
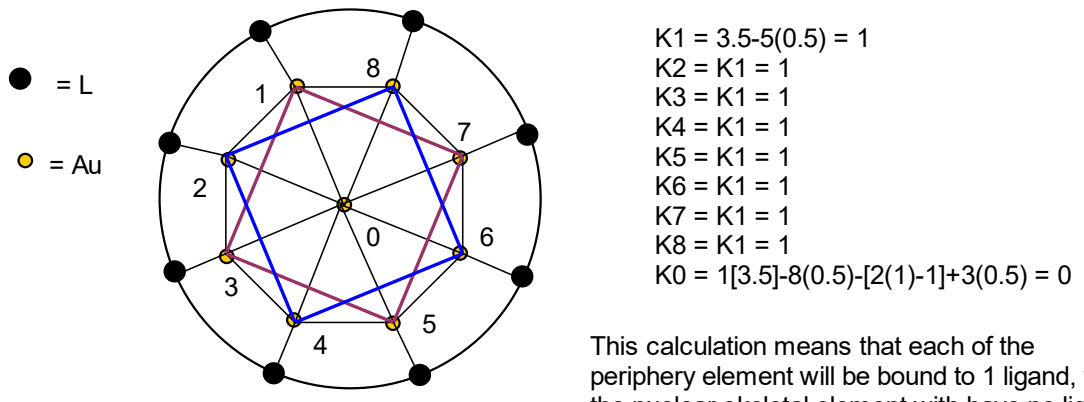

This calculation means that each of the periphery element will be bound to 1 ligand, while the nuclear skeletal element with have no ligand bound to it. 
$\mathrm{Au}_{10} \mathrm{Cl}_{3} \mathrm{~L}_{6}{ }^{+}: \mathrm{K}=10[3.5]-1.5-6+0.5=28 ; \mathrm{K}(\mathrm{n})=28(10) ; \mathrm{S}=4 \mathrm{n}-16, \mathrm{Kp}=\mathrm{C}^{9} \mathrm{C}[\mathrm{M} 1]$

This cluster also has one skeletal element in the nucleus surrounded by 8 other skeletal elements. This is expressed in F-

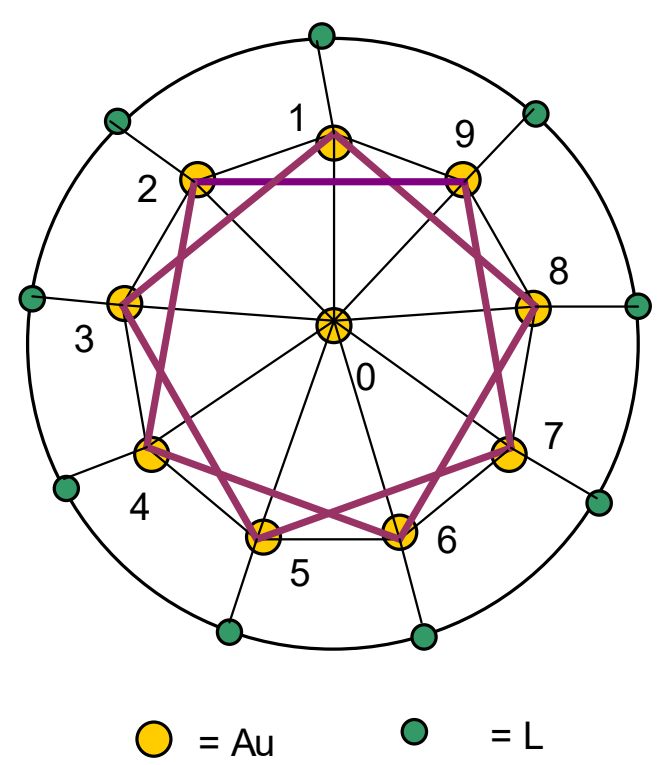

$\mathrm{F}-10$
$\mathrm{K} 1=3.5-2.5=1$

$\mathrm{K} 2=\mathrm{K} 1=1$

$\mathrm{K} 3=\mathrm{K} 1=1$

$\mathrm{K} 4=\mathrm{K} 1=1$

$\mathrm{K} 5=\mathrm{K} 1=1$

$\mathrm{K} 6=\mathrm{K} 1=1$

$\mathrm{K} 7=\mathrm{K} 1=1$

$\mathrm{K} 8=\mathrm{K} 1=1$

$\mathrm{K} 9=\mathrm{K} 1=1$

$\mathrm{K} 0=1[3.5]+0.5-9(0.5)-[2(1)-1]=-1.5$

$\mathrm{NET}=9-1.5=7.5$

This is the same as $6 \mathrm{~L}+3 \mathrm{Cl}$.

The golden clusters such as the ones shown in F-8-f-10 which have a mono-skeletal nuclei are described as TOROIDAL

$\mathrm{Au}_{9} \mathrm{~L}^{+}: \mathrm{K}=9[3.5]-8+0.5=24 ; \mathrm{K}(\mathrm{n})=24(9), \mathrm{S}=4 \mathrm{n}-12, \mathrm{Kp}=\mathrm{C}^{7} \mathrm{C}[\mathrm{M} 2]$

The capping symbol tells us that the cluster will have 2 skeletal elements at the nucleus and will be capped by 7 other skeletal elements. A graph of the skeletal structure can be represented as in F-

$=[\mathrm{M} 2]$ nucleus

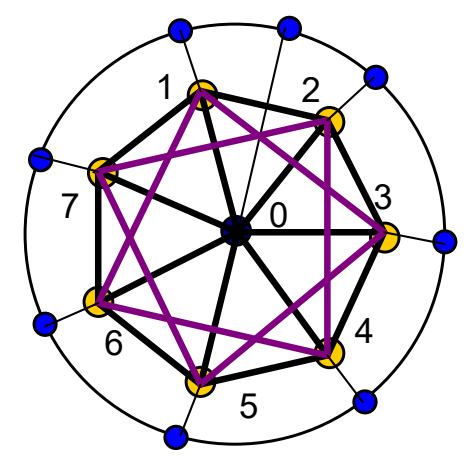

$\mathrm{F}-11$
$\mathrm{K} 1=3.5-2.5=1$

$\mathrm{K} 2=\mathrm{K} 1=1$

$\mathrm{K} 3=\mathrm{K} 1=-1$

$\mathrm{K} 4=\mathrm{K} 1=1$

$\mathrm{K} 5=\mathrm{K} 1=1$

$\mathrm{K} 6=\mathrm{K} 1=1$

$\mathrm{K} 7=\mathrm{K} 1=1$

$\mathrm{K} 0=2[3.5]+0.5-7(0.5)-[2(2)-1]$

$=7 \cdot 5-3 \cdot 5-3=1$

The above simple calculation tells us that each of the periphery skeletal element will bind to one ligand while one of skeletal elements at the nucleus will also bind to one ligand. 


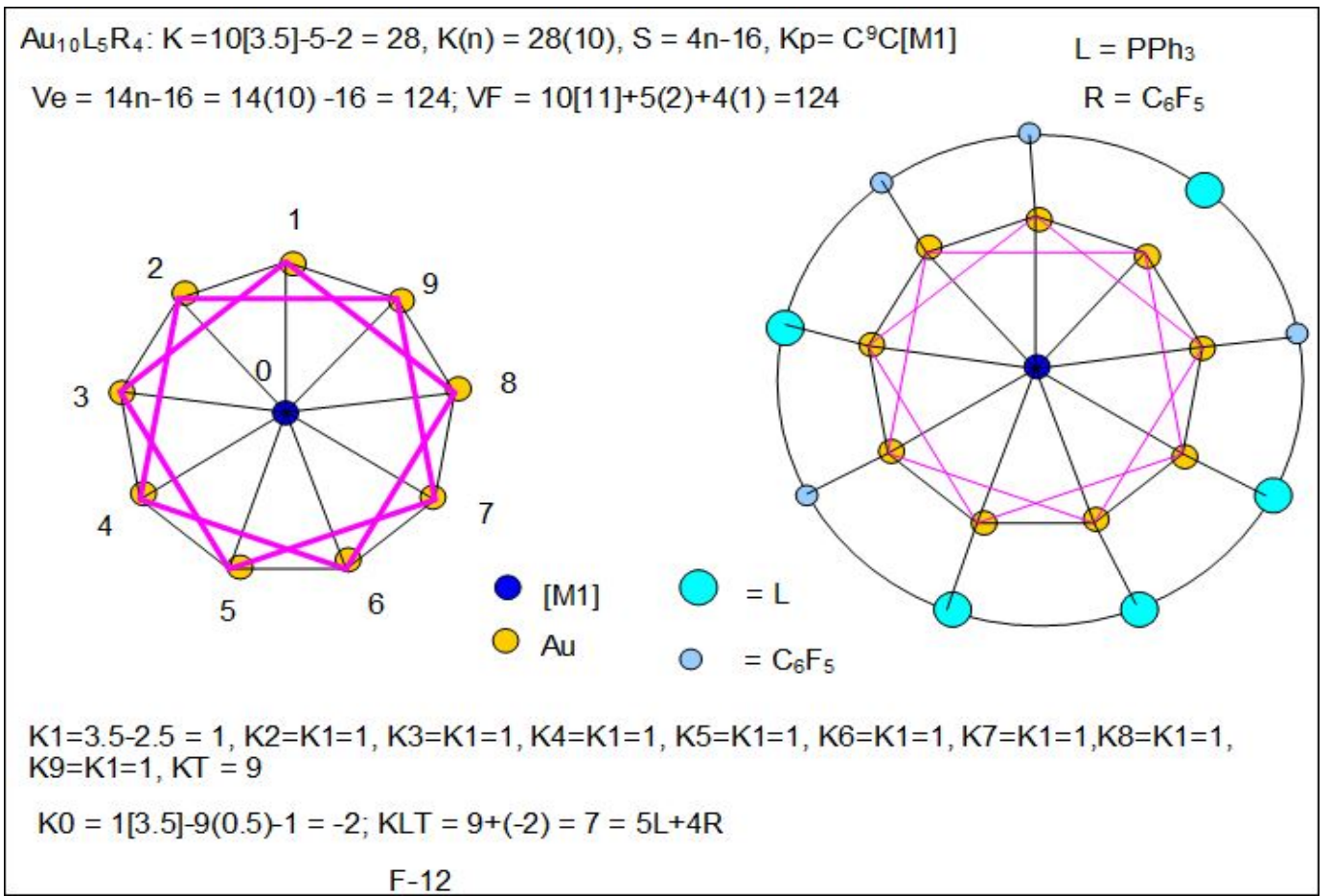

$\mathrm{Au}_{11}(\mathrm{I})_{3} \mathrm{~L} 7: \mathrm{K}=11[3.5]-1.5-7=30(11) ; \mathrm{S}=4 \mathrm{n}-16, \mathrm{Kp}=\mathrm{C}^{9} \mathrm{C}[\mathrm{M} 2]$

This tells us that the cluster has 2 skeletal elements at the nucleus. In other words, it belongs to CLUSTER GROUP 2. The nucleus is also capped by 9 skeletal elements. Its graphical sketch is shown in $\mathrm{F}$ -

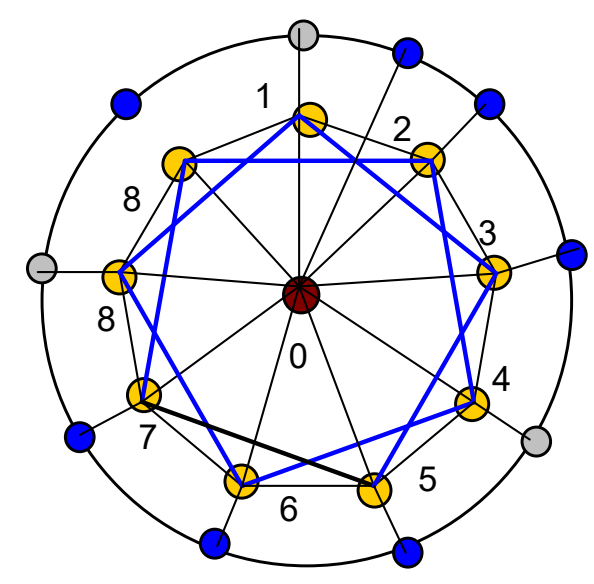

$\mathrm{F}-13$

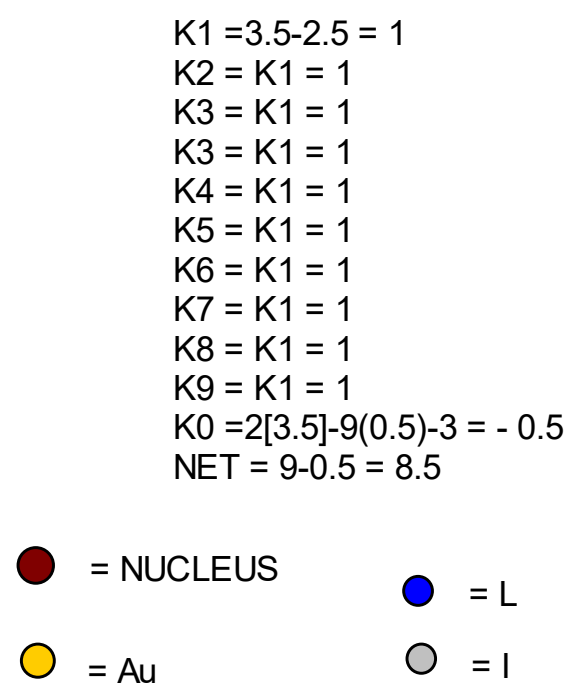

$\mathrm{K} 1=3.5-2.5=1$

$\mathrm{K} 1=1$

$\mathrm{K} 4=\mathrm{K} 1=1$

$\mathrm{K} 5=\mathrm{K} 1=1$

$\mathrm{K} 6=\mathrm{K} 1=1$

$\mathrm{K} 7=\mathrm{K} 1=1$

$\mathrm{K} 8=\mathrm{K} 1=1$

$\mathrm{K} 9=\mathrm{K} 1=1$

政 $=2[3.5]-9(0.5)$

$\mathrm{NET}=9-0.5=8.5$
$\mathrm{O}=$ NUCLEUS
$=\mathrm{Au}$
$0=1$


$\mathrm{Au}_{13} \mathrm{Cl}_{2} \mathrm{~L}_{10}{ }^{3+}: \mathrm{K}=13[3.5]-1-10+1.5=36, \mathrm{~K}(\mathrm{n})=36(13) ; \mathrm{S}=4 \mathrm{n}-20, \mathrm{Kp}=\mathrm{C}^{11} \mathrm{C}[\mathrm{M} 2]$

This means the cluster has 2 skeletal elements at the nucleus while 11 others are capping around the nucleus. This is graphically sketched in F-14.

$$
\begin{aligned}
& \mathrm{K} 1=\mathrm{K} 2=\mathrm{K} 3=\mathrm{K} 4=\mathrm{K} 5=\mathrm{K} 6=\mathrm{K} 7=\mathrm{K} 8=\mathrm{K} 9=\mathrm{K} 10=\mathrm{K} 11=3.5-2.5=1 \\
& \mathrm{~K} 0=2[3.5]-11(0.5)--3+1.5=0
\end{aligned}
$$

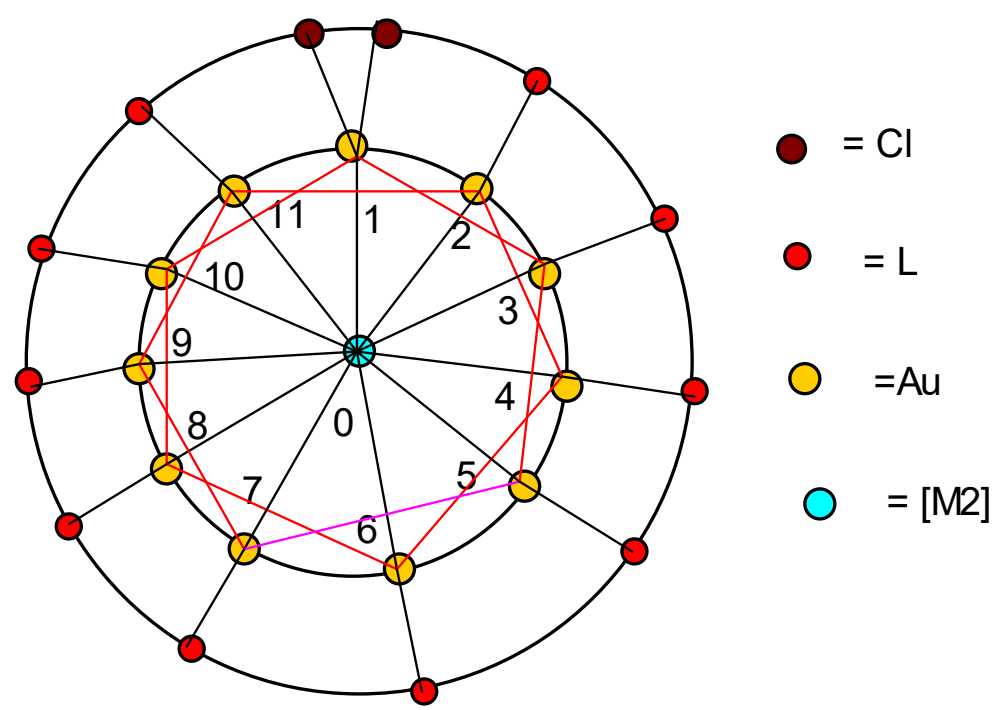

\section{$\mathrm{F}-14$}

The golden clusters with [M2] nuclei are usually described as Spherical (Mingos, 1984; Konishi, 2014; Kiremire, 2016c, 2017a).

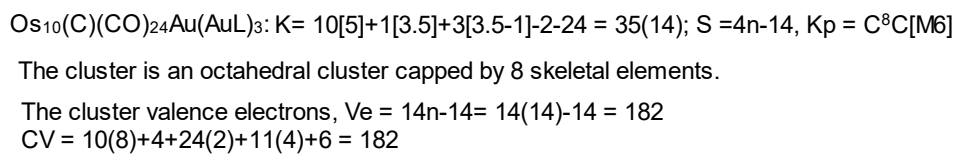

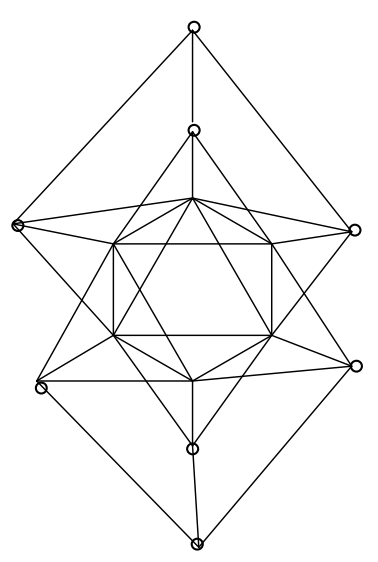

$0=$ Os

$0=\mathrm{Au}$

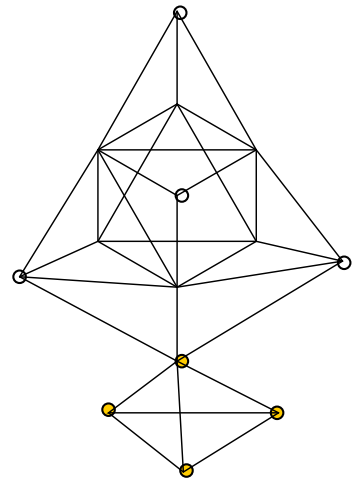

SKETCH OF OBSERVED SKELETAL STRUCTURE

SKELETAL SKETCH OF PREDICTED CLUSTER 


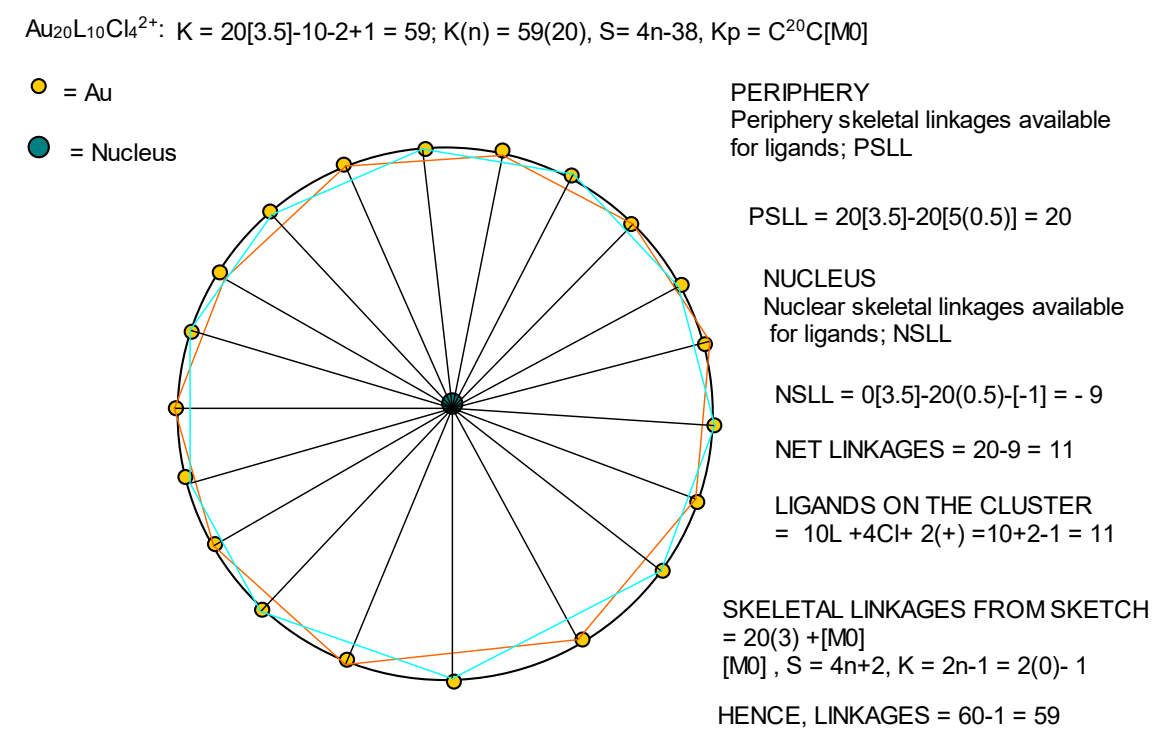

F-16

More information on categorization of clusters including the golden ones can be found in the recently published work(Kiremire, 2018a, 2018b).

APPENDIX-1

\begin{tabular}{|c|c|c|c|c|c|c|c|}
\hline \multicolumn{8}{|c|}{ Skeletal k Values of Main Group Elements } \\
\hline Group & Series, $S=\mathbf{4 n}+q$ & $k=2 n-q / 2$ & & & & & \\
\hline 1 & $4 n-3$ & 3.5 & $\mathbf{L i}$ & $\mathrm{Na}$ & $\mathbf{K}$ & $\mathbf{R b}$ & Cs \\
\hline 2 & $4 n-2$ & 3 & $\mathrm{Be}$ & Mg & $\mathbf{C a}$ & $\mathrm{Sr}$ & Ba \\
\hline 3 & $4 n-1$ & 2.5 & $\mathbf{B}$ & Al & Ga & In & Tl \\
\hline 4 & $4 n+0$ & 2 & $\mathbf{C}$ & $\mathbf{S i}$ & Ge & Sn & $\mathbf{P b}$ \\
\hline 5 & $4 n+1$ & 1.5 & $\mathbf{N}$ & $\mathbf{P}$ & As & Sb & Bi \\
\hline 6 & $4 n+2$ & 1 & $\mathbf{O}$ & $\mathbf{S}$ & Se & $\mathrm{Te}$ & Po \\
\hline 7 & $4 n+3$ & 0.5 & $\mathbf{F}$ & $\mathbf{C l}$ & $\mathrm{Br}$ & I & At \\
\hline 8 & $4 n+4$ & $\mathbf{0}$ & $\mathrm{Ne}$ & Ar & $\mathbf{K r}$ & $\mathrm{Xe}$ & $\mathbf{R n}$ \\
\hline
\end{tabular}

\section{APPENDIX-2}

\section{Conclusion}

\begin{tabular}{|c|c|c|c|c|}
\hline \multicolumn{5}{|c|}{ Skeletal k Values of Transition Metals } \\
\hline 3d-TM* & 4d-TM* & 5d-TM* & Series, $S=\mathbf{4 n}+q$ & $k=2 n-q / 2$ \\
\hline Sc & Y & La & $4 n-11$ & 7.5 \\
\hline Ti & $\mathbf{Z r}$ & Hf & $4 n-10$ & 7 \\
\hline $\mathbf{V}$ & Nb & Ta & $4 n-9$ & 6.5 \\
\hline $\mathrm{Cr}$ & Mo & $\mathbf{W}$ & $4 n-8$ & 6 \\
\hline Mn & Te & $\operatorname{Re}$ & $4 n-7$ & 5.5 \\
\hline $\mathbf{F e}$ & Ru & Os & $4 n-6$ & 5 \\
\hline Co & $\mathbf{R h}$ & Ir & $4 n-5$ & 4.5 \\
\hline $\mathbf{N i}$ & Pd & Pt & $4 n-4$ & 4 \\
\hline $\mathrm{Cu}$ & Ag & Au & $4 n-3$ & 3.5 \\
\hline $\mathbf{Z n}$ & Cd & $\mathrm{Hg}$ & $4 n-2$ & 3 \\
\hline
\end{tabular}

Transition metal cluster formulas can be derived from primary clusters and respective $\mathrm{K}(\mathrm{n})$ parameters. It has been shown that the known clusters can also be derived from the multiples of their respective $\mathrm{K}(\mathrm{n})$ parameters and primary clusters. 


\section{Acknowledgement}

I wish to acknowledge the following hotel for providing material resources to enable complete this work and the immense hospitality extended to me since my arrival in Uganda this this year.

Serene Suites \& Hotel - Mutundwe

Wasswa Micheal Road, Mutundwe

P.O. Box 31654, Mutundwe, Rubaga, Kampala, Uganda, Tel: +256 $312211696,+256757033564,+256752284728$

\section{Dedication}

Dedicated to my beloved dear wife, Merab Kambamu Kiremire who passed on Sunday 21-01-2018, Windhoek, Namibia. Her unwavering support and encouragement of this work will greatly be missed. May her soul rest in eternal peace.

\section{References}

Coenraad, V. D. B. (2006). Aggregation of gold clusters by complementary hydrogen bonding. University of Groningen. Cotton, F. A., \& Wilkinson, G. (1980). Advanced Inorganic Chemistry, $4^{\text {th }}$ Ed., John Wiley and Sons, New York.

Fox, M. A., \& Wade, K. (2003). Evolving Patterns in boron cluster chemistry. Pure Appl. Chem., 75(9), $1315-1323$. https://doi.org/10.1351/pac200375091315

Gimeno, M. C. (2008). The Chemistry of Gold in Modern Supramolecular Gold Chemistry: Gold-Metal Interactions and Applications, Edited, Laguna, A., Wiley-VCH.

Greenwood, N. N., \& Earnshaw, A. (1998). Chemistry of the Elements, $2^{\text {nd }}$ Ed. Butterworth, Oxford.

Housecroft, C. E., \& Sharpe, A. G. (2012). Inorganic Chemistry, $4^{\text {th }}$ Edition, Pearson, Harlow, England

Hughes, A. K., \& Wade, K. (2000). Metal-metal and metal-ligand strengths in metal carbonyl clusters. Coord. Chem. Rev., 197, 191-229. https://doi.org/10.1016/S0010-8545(99)00208-8

Jemmis, E. D. (2005). Building relationships between polyhedral boranes and elemental boron. Inorg. Chem. 18, 620-628.

Jemmis, E. D., \& Prasad, D. L. V. K. (2008). Unknowns in the chemistry of Boron. Current Science, 95(10), 1277-1283

Kiremire, E. M. (2014a). Numerical sequence of Borane Series. Orient. J. Chem., 30(3), 1055-1060. https://doi.org/10.13005/ojc/300317

Kiremire, E. M. (2014b). Validation and verification of the Expanded Table for Transition Metal Carbonyl and Main Group Element Cluster Series which obey the 18-Electron and the 8-Electron Rules Respectively. Orient. J. Chem., 30(4), 1475-1495. https://doi.org/10.13005/ojc/300404

Kiremire, E. M. R. (2016a). The Application of the 4n Series Method to Categorize Metalloboranes. Int. J. Chem., 8(3), 62-73. https://doi.org/10.5539/ijc.v8n3p62

Kiremire, E. M. R. (2016c). Clusters of Gold Containing P-Block Elements. Am. J. Chem., 6(5), 126-144

Kiremire, E. M. R. (2016e). A Hypothetical Model for the Formation of Transition Metal Carbonyl Clusters Based Upon 4n Series Skeletal Numbers. Int. J. Chem., 8(4), 78-110. https://doi.org/10.5539/ijc.v8n4p78

Kiremire, E. M. R. (2017b). The Golden Series and Clusters of Gold-unique Shapes and Bonding. Int. J. Chem., 9(1), 38-57. https://doi.org/10.5539/ijc.v9n1p38

Kiremire, E. M. R. (2017c). Numerical Characterization of Chemical Fragments, Molecules and Clusters Using Skeletal Numbers and Nuclearity Trees. Am. J. Chem., 7(3), 73-96.

Kiremire, E. M. R. (2018a). Graph Theory of Chemical Series and Broad Categorization of Clusters. Int. J. Chem., 10(1), 17-80. https://doi.org/10.5539/ijc.v10n1p17

Kiremire, E. M. R. (2016b). Classification of Zintl Ion Clusters Using 4n Series Approach. Orient. J. Chem., 32(4), 1731-1738. https://doi.org/10.13005/ojc/320401

Kiremire, E. M. R.(2016d). Generating Formulas of Transition Metal Carbonyls Clusters of Osmium, Rhodium, and Rhenium. Int. J. Chem., 8(1), 126-144. https://doi.org/10.5539/ijc.v8n1p126

Kiremire, E. M. R. (2017a). Boranes, Carboranes, Metalloboranes, Transition Metal Carbonyls, and Other Cluster Formulas Obey the Law of Skeletal Numbers and Their Valences. Amer. J. Chem., 7(4), 113-144.

Kiremire, E. M. R. (2018b). Graph Theory of Capping Golden Clusters. Int. J. Chem., 10(1), 87-130. 
https://doi.org/10.5539/ijc.v10n1p87

Konishi, K.(2014). Phosphine-Coordinated Pure-Gold Clusters and Unique Optical Properties /Responses. Structure and Bonding, 161, 49-86. https://doi.org/10.1007/430_2014_143

Lipscomb, W. N. (1963). Boron Hydrides. W. A. Bejamin, Inc., New York.

Luquin, A., Cerrada, E., \& Laguna, M. (2009). Pentafluorophenyl gold complexes. Gold Chemistry, Edited, Mohr, F., Wiley-VCH, Wuppertal, Germany. https://doi.org/10.1002/9783527626724.ch3

Mingos, D. M. P. (1972). A General Theory for Cluster and Ring Compounds of the Main Group and Transition Elements. Nature (London), Phys. Sci., 236, 99-102. https://doi.org/10.1038/physci236099a0

Mingos, D. M. P. (1984). Gold Cluster Compounds: Are they materials in miniature? Gold Bull., 17(1), 5-12. https://doi.org/10.1007/BF03214670

Pauling, L. (1977). Structure of transition metal cluster compounds. Use of an additional orbital resulting from $\mathrm{f}, \mathrm{g}$ character of spd bond orbitals. PNAS, 74(12), 5235-5238. https://doi.org/10.1073/pnas.74.12.5235

Puls, A. B. (2014). A New Synthesis Concept for Metal Doped Gold Clusters. PhD Thesis.

Wade, K. (1971). The structural significance of the number of skeletal bonding electron-pairs in carboranes, the higher boranes and borane ions and various transition metal carbonyl cluster compounds. Chem. Commun., 792-793. https://doi.org/10.1039/c29710000792

\section{Copyrights}

Copyright for this article is retained by the author(s), with first publication rights granted to the journal.

This is an open-access article distributed under the terms and conditions of the Creative Commons Attribution license (http://creativecommons.org/licenses/by/4.0/). 